\title{
Cold-Electron Bolometer
}

\author{
Leonid S. Kuzmin \\ Chalmers University of Technology, \\ Department of Microtechnology and Nanoscience \\ Sweden
}

\section{Introduction}

Cosmic microwave background (CMB) measurements are ranked second by the journal Science among the top 10 Achievements of the Decade. In 2000 and 2003, an experiment known as balloon observations of millimetric extragalactic radiation and geophysics (BOOMERanG) measured the CMB in detail in patches of the sky. Then in 2003, NASA's space-based Wilkinson microwave anisotropy probe (WMAP) mapped the CMB across the sky, producing an exquisite miniature picture of the cosmos. These and the measurements that followed have started transforming cosmology from a largely qualitative endeavour to a precision science with a standard theory, named 'precision cosmology' (Cho, 2010; Masi, 2006). Recent cosmology experiments have discovered that the universe consists mainly of dark energy and dark matter.

Experiments to resolve the nature of these dark components will require a new generation of ultra-sensitive detectors. At present, the most widespread superconducting bolometer is a transition-edge sensor (TES) (Irvin, 1995; Lee et al., 1996). However, due to artificial dc bias heating, the TES has excess noise and strictly limited saturation power.

A novel concept of the Cold-Electron Bolometer (CEB) (Kuzmin, 1998, 2000, 2001, 2004; Kuzmin \& Golubev, 2002) has been invented to overcome these problems. The CEB concept is based on a unique combination of the RF capacitive coupling of an absorber to the antenna through capacitance of the SIN tunnel junctions (Kuzmin, 1998, 2000) and direct electron cooling of the absorber by the same SIN tunnel junction (Kuzmin, 2001; Kuzmin \& Golubev, 2002). The noise properties of this device are improved considerably by decreasing the electron temperature. Direct electron cooling leads also to a considerable increase of the saturation power due to removing incoming power from the sensitive nanoabsorber. Direct electron cooling provides strong negative electrothermal feedback for the signal (Kuzmin, 2004), analogous to the TES (Irvin, 1995). However, the artificial dc heating for feedback in TES is replaced by using an effect of electron cooling by SIN tunnel junctions (Nahum 1994) for direct electron cooling of the absorber by SIN tunnel junctions (Kuzmin 1998; Golubev \& Kuzmin, 2001) to a minimum temperature (that could be less than phonon temperature). This concept can lead to a major breakthrough in the realisation of supersensitive detectors.

Historically, development of SIN tunnel junctions as a thermometer was started in the 1970s (Bakker et al., 1970). The SIN junction demonstrated sensitivity to the temperature of a normal 
metal. A normal metal hot-electron bolometer (NHEB) with SN Andreev mirrors for thermal isolation and a SIN tunnel junction for readout was proposed in 1993 (Nahum et al., 1993) and realized in the same year (Nahum \& Martinis, 1993). Disadvantages of the NHEB are:

- quite a small dynamic range due to low saturation power for the ultrasensitive bolometer with a small absorber (SIN junction working as a voltmeter)

- poisoning of the bolometer by quasiparticles due to entrapping through Andreev contacts (Kuzmin et al., 1999)

- $\quad$ very complicated RF structure is disturbed by a central electrode for dc readout; in particular, it is impossible to create an array of bolometers (the most advanced concept of CEBs for the moment).

The effect of electron microrefrigeration by SIN tunnel junction was demonstrated in 1994 (Nahum et al., 1994). Quite effective cooling of the small absorber from 300 to $100 \mathrm{mK}$ was demonstrated in several groups (Leivo et al., 1996; Kuzmin et al., 2004). Then several groups moved in the direction of using this microrefrigeration for cooling the platform with the detectors placed on this platform. However, this direction did not show good progress because of dramatic difficulties in cooling at low temperatures due to weak electron-phonon interaction and the fact that it only influenced the electron-phonon noise component. The main component, background noise, was not influenced by electron cooling.

A decisive step in the development of superconducting detectors has been the invention of a transition-edge sensor (TES) with strong electrothermal feedback (Irvin, 1995; Lee et al., 1996). However, the TES has some problems with excess noise, saturation and the most dramatic problem of artificial overheating by dc power for the electrothermal feedback. This unavoidable additional heating kills all efforts on deep cooling and does not give good prospects for the realisation of ultimate performance of the bolometer.

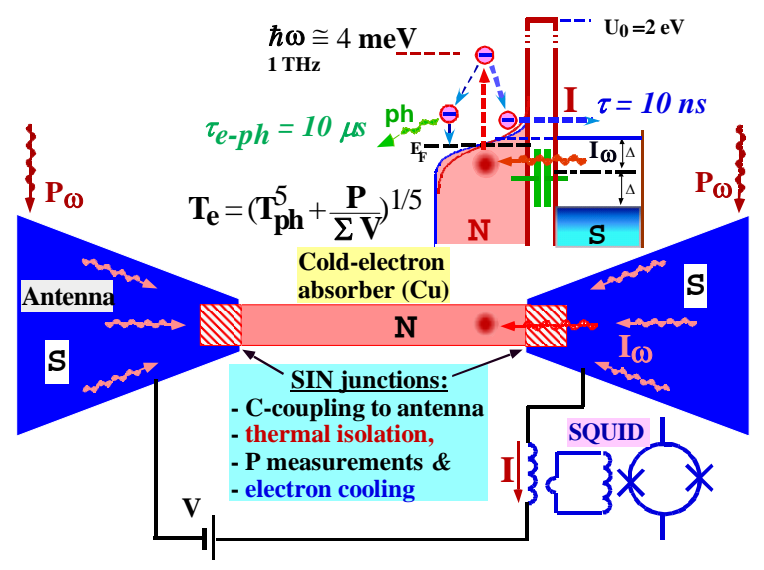

Fig. 1. Capacitively coupled cold-electron bolometer with nanoabsorber and SIN tunnel junctions for direct electron cooling and power measurements. The signal power is supplied to the sensor through capacitance of tunnel junctions, dissipated in the nanoabsorber and removed back from the absorber as hot electrons by the same SIN junctions. The electron cooling serves as strong negative electrothermal feedback improving all characteristics of the CEB: time constant, responsivity and NEP. 
In contrast to this overheating, a new concept of a CEB with direct electron cooling has been proposed by Kuzmin (Kuzmin, 1998, 2000, 2001, 2004; Kuzmin \& Golubev, 2002). The CEB is the only concept removing incoming background power from the supersensitive region of absorber. The CEB avoids the main problem of TES, an additional dc heating for the electrothermal feedback, by replacing it with the direct electron cooling of the absorber that could be a turning point in the realisation of modern supersensitive detectors. This cooling could be especially important for the realisation of high sensitivity in the presence of the realistic background power load because it returns the system to lowest temperature (noise) state. It could help to avoid full saturation when the signal exceeds the level of dc bias power that is the great problem for the TES. The CEB could give a high dynamic range in combination with the SQUID readout system having a high dynamic range in closed-loop operation. In this state the system shows the most reaction (responsivity) to an incoming signal. All power of the signal is used for measurements. A possible objection that tunnelling of electrons would increase shot noise is rejected by simple argument: if power is not removed by tunnel junctions, the same type of shot noise will be created by phonons through increased electron-phonon interaction.

\section{Main concepts of the Cold-Electron Bolometer (CEB)}

The basic concept of the CEB is a cold-electron bolometer with capacitive coupling to the antenna by two SIN tunnel junctions (Kuzmin, 2000, 2001; Kuzmin \& Golubev, 2002). Theoretical estimations and preliminary experiments show that it is possible to realize the necessary sensitivity of better than $10^{-18} \mathrm{~W} / \mathrm{Hz}^{1 / 2}$ with antenna-coupled nanobolometers at a temperature of $\leq 0.3 \mathrm{~K}$. Additional advantages of such detectors are the possibility to operate in a wide range of background load, easy integration in arrays on planar Si substrate and the possibility of polarisation measurements. Flexibility of the CEB concept gives the opportunity to realise this bolometer for any power load from $0.02 \mathrm{fW}$ for space applications to $10 \mathrm{pW}$ for ground-based applications with NEP less than photon noise of the signal.

To match the CEB with the requested JFET readout, two novel concepts of CEB have been proposed: parallel/series array of CEBs and 2D focal plane array of CEBs with SIN tunnel junctions. Simulations show high performance of these bolometers with NEP less than photon noise of the signal. These concepts should be qualified experimentally in RF tests and implemented in BOOMERanG. These concepts could also be used in OLIMPO, SPICA, B-Pol and Millimetron cosmology instruments.

\subsection{General theory of the CEB}

The operation of CEB can be analysed using a heat balance equation (Golubev \& Kuzmin, 2001; Kuzmin \& Golubev, 2002)

$$
P_{S I N}\left(V, T_{e}, T_{p h}\right)+\Sigma \Lambda\left(T_{e}^{5}-T_{p h}^{5}\right)+C_{\Lambda} \frac{d T}{d t}=P_{0}+\delta P(t)
$$

Here, $\Sigma \Lambda\left(T_{e}^{5}-T_{p h}^{5}\right)$ is the heat flow from electron to the phonon subsystems in the normal metal, $\Sigma$ is a material constant, $\Lambda$ - a volume of the absorber, $T_{e}$ and $T_{p h}$ are, respectively, the electron and phonon temperatures of the absorber; $\mathrm{P}_{\mathrm{SIN}}\left(\mathrm{V}, \mathrm{T}_{\mathrm{e}}, \mathrm{T}_{\mathrm{ph}}\right)$ is cooling power of the SIN 
tunnel junctions; $C_{v}=\gamma T_{e}$ is the specific heat capacity of the normal metal and $P(t)$ is the incoming $\mathrm{RF}$ power. We can separate Eq. (1) into the time independent equation, $\Sigma \Lambda\left(T_{e 0}^{5}-T_{p h}^{5}\right)+P_{S I N 0}\left(V, T_{e 0}, T_{p h}\right)=P_{0}$, and the time dependent equation,

$$
\left(\frac{\partial P_{S I N}}{\partial T}+5 \Sigma \Lambda T_{e}^{4}+i \omega C_{\Lambda}\right) \delta T=\delta P
$$

The first term, $G_{S I N}=\partial P_{S I N} / \partial T$, is the cooling thermal conductance of the SIN junction that gives the negative electrothermal feedback (ETF); when it is large, it reduces the temperature response $\delta T$ because cooling power, $P_{S I N}$, compensates the change of signal power in the bolometer. The second, $G_{e-p h}=5 \Sigma \Lambda T_{e}^{4}$, is electron-phonon thermal conductance of the absorber. From Eq. (2) we define an effective complex thermal conductance which controls the temperature response of $\mathrm{CEB}$ to the incident signal power

$$
G_{e f f}=G_{c o l}+G_{e-p h}+i \omega C_{\Lambda}
$$

Full expression for current is

$$
I=\frac{1}{e R} \int d E N s(E)[f(E, T e)-f(E-e V, T p h)]+\frac{V}{R j}
$$

where $\mathrm{Ns}(\mathrm{E})=|\mathrm{E}| / \sqrt{ }\left(\mathrm{E}^{2}-\Delta^{2}\right)$ is the normalized density of states in the superconductor and $\mathrm{f}(\mathrm{E}, \mathrm{T})=1 /[\exp (\mathrm{E} / \mathrm{T})+1]$.

In analogy with TES (Irvin, 1995; Lee et al.,1996), the effective thermal conductance of the CEB is increased by the effect of electron cooling (negative ETF).

The current responsivity is given by

$$
S_{i}=\frac{\partial I}{\partial P}=\frac{\partial I / \partial T}{G_{c o o l}+G_{e-p h}+i \omega C_{\Lambda}}=\frac{\partial I / \partial T}{G_{c o o l}} \frac{L}{(L+1)[1+i \omega \tau]},
$$

where $L=G_{\text {cool }} / G_{e-p h}>>1$ is ETF gain and

$$
\tau=C_{\Lambda} / G_{e-p h}=\tau_{0} /(L+1)
$$

is an effective time constant, $\tau_{0}=C_{\Lambda} / G_{e-p h}(\cong 10 \mu \mathrm{s}$ at $100 \mathrm{mK})$.

Strength of electrothermal feedback is estimated as:

$$
L(\omega)=\frac{G_{c o o l}}{G_{e-p h}(1+i \omega \tau)}=\frac{\partial I / \partial T}{G_{c o o l}+G_{e-p h}+i \omega C_{\Lambda}}
$$

\subsubsection{CEB and TES comparison}

We compare now the realization of CEB and TES. The principle of operation is shown in Fig. 3. Both concepts use the voltage-biased mode of operation. The TES is heated to critical 
temperature by dc power $\mathrm{P}_{\text {bias }}$ (Fig. 2a). This temperature is supported during all range of operation (before saturation). Electrothermal feedback arises from the dependence of the bias power on the resistance of the superconductor. If there is an increase in optical power incident on the bolometer, the bias power decreases and nearly compensates for the increase incident power (Fig. 2b). Output signal is this decrease in bias power nearly equals the incident power.

The principle of operation of the CEB (Fig. 2a) is approximately the same, but moving to another bias point in temperature: to absolute zero. Starting from the phonon temperature $\mathrm{T}_{\mathrm{ph}}=100 \mathrm{mK}$, the cooling conductance, $\mathrm{G}_{\mathrm{cool}}$, decrease the electron temperature to the possible minimum level, $30 \mathrm{mK}$ in this case (for typical parameters of CEB: $\Lambda=0.01 \mu \mathrm{m}^{3}$ and $\mathrm{R}=1 \mathrm{k} \Omega$ ). After applying signal power, the cooling conductance increases trying to compensate for the increase in the electron temperature to the minimum temperature close to the previous value.

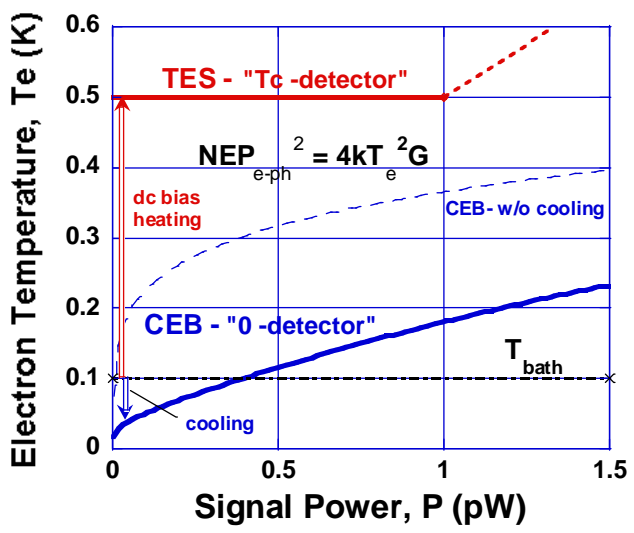

(a)

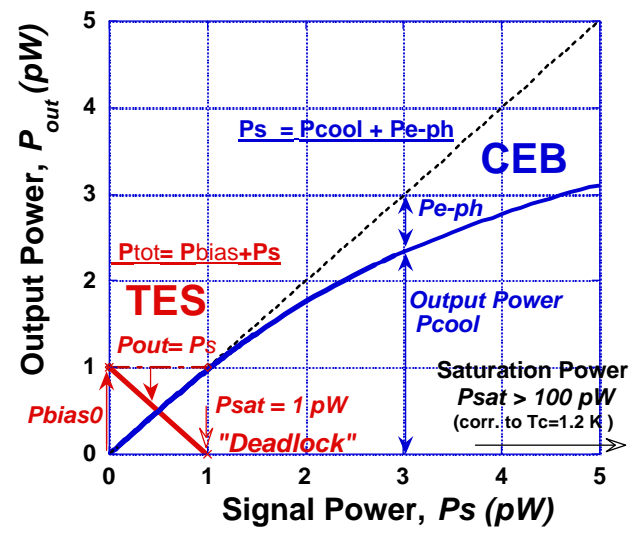

(b)

Fig. 2. (a) Electron temperature as a function of signal power for $T_{\text {bath }}=100 \mathrm{mK}$ for $C E B$ and TES. For CEB, the $T_{e}$ is always cooled to the lowest possible level. For $P<0.4 p W$, the $T_{e}$ of CEB is less than $\mathrm{T}_{\text {bath }}$ (real cold-electron bolometer). For TES, the $\mathrm{T}_{\mathrm{e}}$ is equal to $\mathrm{T}_{\mathrm{C}}$ for all range of operation up to saturation power. After saturation there is uncontrollable increase of temperature. (b) Output power (cooling power) of the CEB in dependence on signal power (blue). They are almost equal at a small level of power. At a higher level of power, the P is split between Pcool and Pe-ph. The saturation power would be achieved only after heating to $\mathrm{Tc}$ of $\mathrm{Al}$ electrode $\left(\mathrm{P}_{\text {sat }}\right.$ is around $\left.100 \mathrm{pW}\right)$. Bias power and output power of the TES on signal power (red). Saturation power is equal to the full bias power at zero signal.

Dependence of output power on signal power is shown in Fig. 2b. For both concepts, Pout is nearly equal to incoming power in the range of dc heating power (TES) and typical cooling power (CEB). Accuracy of removing (CEB) or compensation (TES) of incoming power is determined by the strength of the electrothermal feedback - loop gain L. For TES, the L is determined by nonlinearity of $\mathrm{R}(\mathrm{T})$ dependence and could exceed 1000 . For $\mathrm{CEB}$, the $\mathrm{L}$ is determined as relation of thermal conductances (6). Typical dependence of $\mathrm{L}$ on incoming power is shown in Fig. 3. 


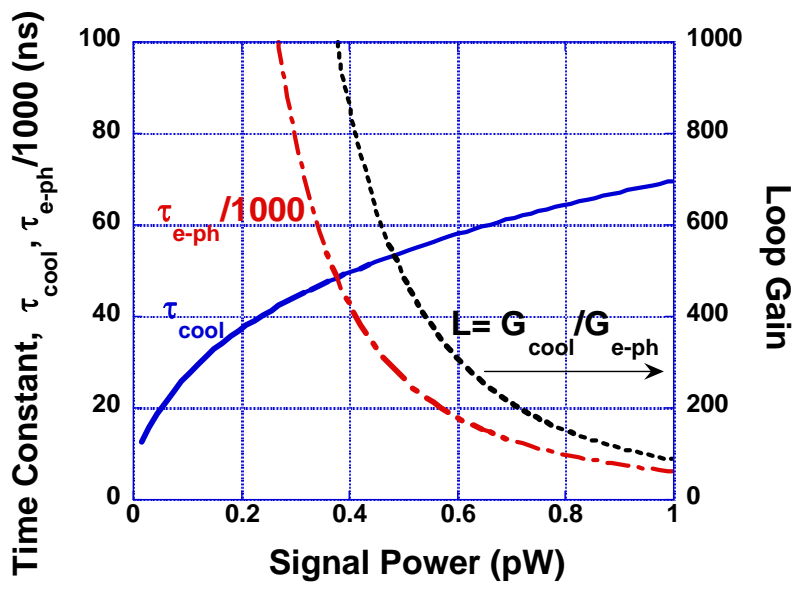

Fig. 3. Time constant of CEB, $\tau_{c o o l}$, in dependence on signal power $P_{s}$. The electron-phonon time constant, $\tau_{e-p h}$, is shown for comparison (scaled 1000 times). The $\tau_{\text {cool }}$ is considerably shorter than $\tau_{e-p h}$ and difference is increased when we move to smaller signal power with stronger electron cooling.

The saturation problem is very serious for TES: $P_{\text {sat }}$ is exactly equal to applied dc heating power $\mathrm{P}_{\text {bias }}$ (Fig. 2b). If we increase saturation level, the overheating of the TES would unavoidably be increased. After saturation power, the TES stops operation fully. It is difficult to foresee the expected level of maximum power load and choosing $\mathrm{P}_{\text {sat }}$ is a really complicated problem.

The situation for CEB saturation is completely different. There is no dramatic problem at all: the output cooling power would simply deviate from the linear dependence Pcool(P). For the typical cooling power around $1 \mathrm{pW}$, the deviation from linear dependence $\mathrm{P}_{\text {out }}(\mathrm{P})$ would be only several \% at this level (Fig. 2b). As signal power is further increased, the deviation will be larger, but the CEB still continues to work. It is only a question of calibration of this dependence. Final 'deadlock' for the CEB would be at the level of power around $100 \mathrm{pW}$ when temperature achieves the critical temperature of the Al electrode. Thus, due to absence of artificial heating, the CEB does not really have the saturation problem and can considerably extend possible scope of operation.

\subsubsection{Time constant}

The time response of the CEB (6) in dependence on incoming power is shown in Fig. 3. As for TES, it is strongly reduced by loop gain L (7) of electrothermal feedback. Cooling conductance $\mathrm{G}_{\mathrm{cool}}$ is not dependent strongly on incoming power and slightly reduced for smaller power. In contrast, the e-ph conductance, $\mathrm{G}_{\mathrm{e}-\mathrm{ph}}$, is very dependent on power due to $4^{\text {th }}$ dependence on electron temperature and strongly increased for low power as well as related e-ph time constant. As a final result, the $\mathrm{L}$ is considerably increased for small power and exceeds the level of 1000 .

The main characteristic of negative electrothermal feedback, the loop gain $L$, is shown by the dashed line. The loop gain is strongly increased for smaller $P_{s}$ due completely to the decrease of $G_{e-p h}$. 


\subsubsection{Noise Properties (NEP)}

Noise properties are characterized by the noise equivalent power (NEP), which is the sum of three different contributions and is defined as follows:

$$
N E P_{\text {total }}^{2}=N E P_{e-p h}^{2}+N E P_{S I N}^{2}+\frac{\delta I^{2}}{S_{I}{ }^{2}} .
$$

Here

$$
N E P_{e-p h}^{2}=10 k_{B} \Sigma \Lambda\left(T_{e}^{6}+T_{p h}^{6}\right)
$$

is the noise associated with electron-phonon interaction (Golwala, S. et al., 1997; Golubev \& Kuzmin, 2001) $N E P^{2}$ SIN is the noise of the NIS tunnel junctions and the last term $\delta I^{2} / S^{2} I_{I}$ is due to the finite sensitivity of the amplifier (SQUID) $\delta I$, which is expressed in $\mathrm{pA} / \mathrm{Hz}^{1 / 2}$.

The noise of the SIN tunnel junctions, $N E P^{2}{ }_{S I N}$, has three components: shot noise $2 e I / S^{2}{ }_{I}$, the fluctuations of the heat flow through the tunnel junctions and the correlation between these two processes

$$
N E P_{S I N}^{2}=\frac{\delta I_{\omega}^{2}}{S_{I}^{2}}-2 \frac{<\delta P_{\omega} \delta I_{\omega}>}{S_{I}}+\delta P_{\omega}^{2} .
$$

Due to this correlation the short noise is decreased at $30-70 \%$. A similar correlation can be found in TES decreasing Johnson noise.

\subsubsection{Ultimate noise performance of the CEB. General limit noise formula}

This question about ultimate noise performance has arisen in relation to the highest requirements placed on NEP for future space missions. The question is, how realistic are these requirements on $\mathrm{NEP}=10^{-20} \mathrm{~W} / \mathrm{Hz}^{1 / 2}$ ?

Ultimate performance of CEB and other bolometers has been analysed. Photon noise is not included in this analysis and should be added later as additional external noise. The NEP is determined by the shot noise due to the power load. The shot noise is treated in a general sense, including e-ph shot noise, due to the emission of phonons. Other sources of noise are neglected due to small values. For the level of $\mathrm{P}_{0}=10 \mathrm{fW}$, this limit can be achieved using relatively low temperatures $(\sim 100 \mathrm{mK})$ and a small volume of the absorber $\left(\Lambda \leq 0.003 \mu \mathrm{m}^{3}\right)$ when we can neglect the electron-phonon noise component.

The general ultimate NEP formula for shot noise limitation has been derived (Kuzmin, 2004):

$$
N E P_{\text {shot }}=\left(2 P_{0} E_{\text {quant }}\right)^{1 / 2}
$$

where $\boldsymbol{P}_{\mathbf{0}}$ - background power load, and $\boldsymbol{E}_{\text {quant }}$-energy level of $\boldsymbol{P}_{\mathbf{0}}$ quantization:

$E_{\text {quant }}=k_{\mathrm{B}} T_{e}$-for normal metal absorber,

$E_{\text {quant }}=\Delta-$ for superconducting absorber.

Ultimate NEP can be estimated for different bolometers for relatively low power load $P_{0}=10 f W$ : 


\begin{tabular}{|c|c|c|c|c|}
\hline $\begin{array}{l}\text { Type of } \\
\text { bolometer }\end{array}$ & $\begin{array}{c}\text { Characteristic } \\
\text { parameter of } \\
\text { absorber }\end{array}$ & $\begin{array}{l}\text { 2. Energy of } \\
\text { quantization }\end{array}$ & $\begin{array}{l}\text { 3. Quantum } \\
\text { efficiency } \\
\hbar \omega / \mathrm{E}_{\text {quant }}\end{array}$ & $\mathbf{N E P}_{\text {shot }}$ \\
\hline CEB & $\mathrm{T}_{\mathrm{e}}=50 \mathrm{mK}$ & $\mathrm{k}_{\mathrm{B}} \mathrm{T}_{\mathrm{e}}=4.3 \mu \mathrm{eV}$ & 950 & $1^{*} 10^{-19} \mathrm{~W} / \mathrm{Hz}^{1 / 2}$ \\
\hline TES & $\mathrm{T}_{\mathrm{C}}=500 \mathrm{mK}$ & $\Delta=73 \mu \mathrm{eV}$ & 56 & $4^{*} 10^{-19} \mathrm{~W} / \mathrm{Hz}^{1 / 2}$ \\
\hline KID & $\mathrm{T}_{\mathrm{C}}=1.2 \mathrm{~K}(\mathrm{Al})$ & $\Delta=200 \mu \mathrm{eV}$ & 20 & $7^{*} 10^{-19} \mathrm{~W} / \mathrm{Hz}^{1 / 2}$ \\
\hline
\end{tabular}

Table 1.

The lowest NEP can be achieved for CEB with the lowest level of quantization. However, even these extreme parameters of $\boldsymbol{P}_{0}$ and $\boldsymbol{E}_{\text {quant }}$ show that it is rather unrealistic to achieve $\mathrm{NEP}=10^{-20} \mathrm{~W} / \mathrm{Hz}^{1 / 2}$ for $\boldsymbol{P}_{0}=10 \mathrm{fW}$.

Systems with linear on $\mathrm{T}$ thermal conductance

- $\quad$ Spider-web TES with conductance through the legs,

- $\quad$ CEB with cooling through SIN tunnel junctions (weak dependence on T: G $\sim T^{1 / 2}$ ).

Limit shot noise is described by general formula (9) with numerical coefficient 2.

Systems with dominant e-ph thermal conductance (strong nonlinearity on T: $\mathrm{G}_{\mathrm{e}-\mathrm{ph}} \sim \mathrm{T}^{4}$ )

- All bolometers on plane substrates with e-ph conductance.

- $\quad$ Antenna-coupled TES on chip with Andreev mirrors.

- $\quad$ NHEB with Andreev mirrors.

Due to strong nonlinearity of e-ph conductance, the limit shot noise is described by modified general formula (9) with five times increased coefficient 10.

As a common conclusion, if we leave the system for normal relaxation of energy through eph interaction, the shot noise is increased due to strong nonlinear dependence of electronphonon thermal conductance on temperature in contrast to linear systems with weak dependence on temperature (or absence of it). This general formula (9) can be effectively used for estimation of the ultimate parameters of CEB and other bolometers for the given parameters of detector systems.

\subsection{CEB in current-biased mode}

\subsubsection{Parallel/series array of CEBs with JFET readout}

A novel concept of the parallel/series array of CEBs with superconductor-insulator-normal (SIN) tunnel junctions has been proposed for work under relatively high optical power load $(10 \mathrm{pW})$ and matching with the JFET readout (Kuzmin 2008a, 2008b) (Fig. 2). The currentbiased CEBs are connected in series for dc and in parallel for HF signals. A signal is concentrated to the absorber through the capacitance of tunnel junctions and additional capacitance for coupling of superconducting islands. Due to the division of power between CEBs in the array and increasing responsivity, the noise matching could be effectively optimised and the photon noise equivalent power could be easily achieved at $300 \mathrm{mK}$ with a room temperature JFET readout.

The CEB concept has been accepted as the main detector for the $350 \mathrm{GHz}$ channel of BOOMERanG (Masi S. et al., 2006). The main requirement is to develop a CEB array with a JFET readout for the 90 channel system. The NEP of the CEB should be less than photon 
noise for optical power load of $10 \mathrm{pW}$ and polarisation resolution should be better than 25 $\mathrm{dB}$ for observations of the $\mathrm{CMB}$ foreground polarisation.

The main innovation of the CEB array in comparison with a single CEB is the distribution of power between $\mathrm{N}$ series CEBs, summarising the increased response from the array. Effective distribution of power is achieved by a parallel connection of CEBs, which is coupled to the RF signal through additional capacitances (Fig. 4). The response is increased because the CEB is sensitive to the level of power and the power is decreased $\mathrm{N}$ times for the individual CEBs, with a proportional decrease of absorber overheating.

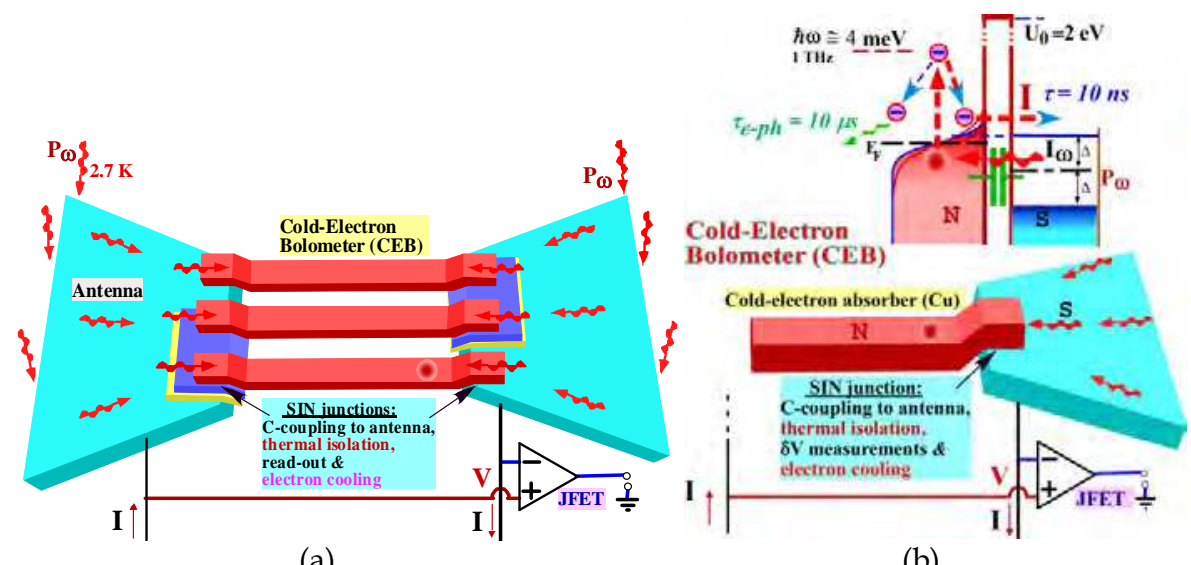

(a)

(b)

Fig. 4. (a) Schematic of a parallel/series array of CEBs with SIN tunnel junctions and JFET readout for the CMB polarization measurements. The current-biased CEBs are connected in series for dc and in parallel for HF signals through the capacitance of a SIN tunnel junction and additional capacitances; (b) The SIN tunnel junction is used also for electron cooling and for reading out the signal with a JFET.

The main purpose of this concept is to match the total dynamic resistance of the array to the noise impedance of a JFET $(\sim 0.6 \mathrm{M} \Omega)$. The power should be divided between the CEBs in the array to increase the responsivity due to lower overheating and moderate electron cooling. The high noise impedance of a JFET amplifier is one of the reasons why a lowohmic TES (Irvin, 1995; Lee et al.,1996) could not be used for this application.

The operation of a CEB array can be analysed using the heat balance equation for a single CEB (1) taking into account power distribution between $\mathrm{N}$ bolometers:

$$
\Sigma \Lambda\left(T_{e}^{5}-T_{p h}^{5}\right)+P_{S I N}\left(V, T_{e}, T_{p h}\right)+C_{v} \frac{d T_{e}}{d t}=\frac{P_{0}+\delta P(t)}{N}+2 \frac{V^{2}}{R_{S}}+I^{2} R_{A}
$$

A bolometer is characterized by its responsivity, noise equivalent power and the time constant. In the current-biased mode, the responsivity, $S_{V}$, is described by the voltage response to an incoming power

$$
S_{V}=\frac{\delta V_{\omega}}{\delta P_{\omega}}=\frac{\partial V / \partial T}{G_{e-p h}+2 G_{S I N}+i \omega C_{\Lambda}}
$$


Noise properties are characterized by the noise equivalent power (NEP), which is the sum of three contributions. For a series array of CEBs, the NEP is defined as follows:

$$
N E P_{\text {tot }}^{2}=N^{*} N E P_{e-p h}^{2}+N^{*} N E P_{S I N}^{2}+N E P_{J F E T}^{2}
$$

Here $N E P_{\text {e-ph }}$ is the noise associated with electron-phonon interaction (9); $N E P_{S I N}$ is the noise of the SIN tunnel junctions. The SIN noise has three components: the shot noise $2 \mathrm{eI} / \mathrm{S}_{\mathrm{I}}$, the fluctuations of the heat flow through the tunnel junctions and the correlation between these two processes (Golwala, S. et al., 1997; Golubev \& Kuzmin, 2001):

$$
N E P_{S I N}^{2}=\frac{\delta I_{\omega}^{2}}{\left(\frac{\partial I}{\partial V} S_{V}\right)^{2}}+2 \frac{<\delta P_{\omega} \delta I_{\omega}>}{\frac{\partial I}{\partial V} S_{V}}+\delta P_{\omega}^{2} .
$$

This correlation is a form of the electrothermal feedback discussed earlier by Mather (Mather, 1982). Due to this correlation the shot noise is increased at $30-50 \%$ in contrast to the SCEB in voltage-biased mode where strong anti-correlation decreases the shot noise (Golubev \& Kuzmin, 2002).

The last term is due to the voltage $\delta V$ and current $\delta I$ noise of the amplifier (JFET), which are expressed in $\mathrm{nV} / \mathrm{Hz}^{1 / 2}$ and $\mathrm{pA} / \mathrm{Hz}^{1 / 2}$ :

$$
N E P_{J F E T}^{2}=\frac{\delta V^{2}+\left(\delta I *(2 R d+R a)^{*} N\right)^{2}}{S_{V}{ }^{2}}
$$

The strong dependence on $\mathrm{N}$, decreasing this noise is included in the responsivity $S_{V}$, which is proportional to the $\mathrm{N}$.

The proposed mode of CEB operation is a current-biased array with voltage readout by a JFET amplifier. The analysis of a single current-biased CEB with JFET readout has shown that there is no chance to get down to photon noise level for high power load due to decreased responsivity and JFET voltage noise (Kuzmin, 2008a, 2008b). The main reason is degradation of voltage responsivity under high optical power load due to overheating of the absorber.

The only chance to achieve a photon noise level is to use a series dc connection of bolometers. However, series HF connections of $\mathrm{N}$ bolometers would lead to real problems of junction size (capacitance should be increased proportionally to N) and overheating of islands. A special innovation has been proposed to combine these requirements: series connection for dc and parallel connection for RF. It could be realized by using additional capacitances for the HF coupling as shown in Figure 2(b). In this case, the input power is divided between bolometers, the electron temperature is decreased and the CEBs increase responsivity while the output signal is collected from all bolometers.

For power load of $P 0=5 \mathrm{pW}$ per polarization, the photon noise could be estimated as

$$
N E P_{\text {phot }}=\sqrt{2 P_{0}{ }^{*} h f}=4.3 * 10^{-17} \mathrm{~W} / \mathrm{Hz}{ }^{1 / 2} \text {. }
$$


We have simulated arrays of CEBs with different numbers of CEBs, from 1 to 26, to achieve a low NEP with JFET readout. The dependence of the noise components on the number of bolometers is shown in Figure 5. The total NEP decreases to a level less than photon noise for a number of CEBs larger than six (three for each probe). It is achieved mainly through the suppression of the JFET noise due to increased responsivity. Figure 5 demonstrates a strong linear increase of the responsivity proportional to $\mathrm{N}$ when the number of bolometers is increased. This dependence is well supported by linear asymptotic. The noise of the JFET is proportionally decreased, which is the main goal of this realization. Around the optimum point $(\mathrm{N}=22)$ the $\mathrm{NEP}_{\text {JFET }}$ is less than $\mathrm{NEP}_{\mathrm{SIN}}$, which is a manifestation of backgroundlimited operation. The $\mathrm{NEP}_{\text {SIN }}$ increases proportionally to $\sqrt{\mathrm{N}}$, but decreases due to decrease of the heat flow (and current) and an increase of the responsivity S. These two effects approximately compensate each other and $\mathrm{NEP}_{\text {SIN }}$ is not sensitive to the number of the bolometers. The most surprising result is that the $\mathrm{NEP}_{\text {eph }}(9)$ is not increased proportionally to the number of bolometers when the total volume of the absorber is increased proportionally to $\mathrm{N}$. This is due to a compensation of this dependence by some decrease in $T_{\mathrm{e}}$ that is in the $6^{\text {th }}$ power for NEPeph (9).

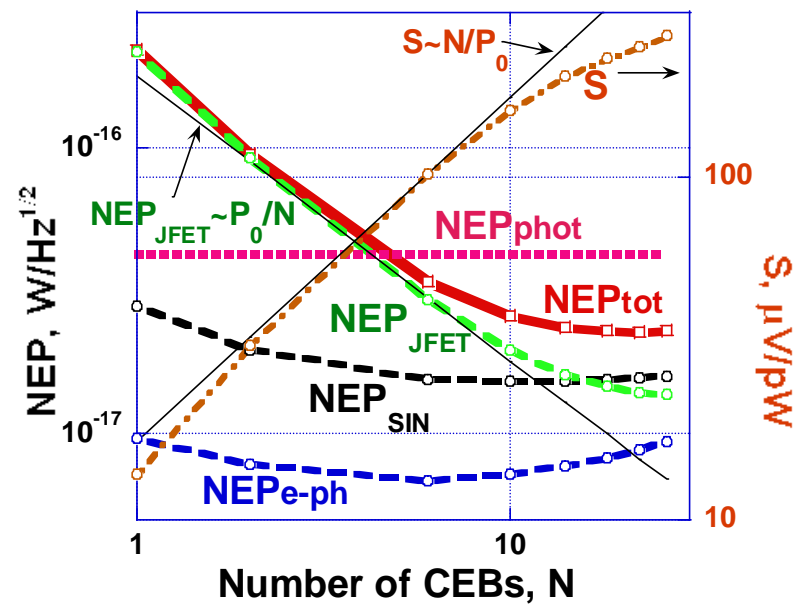

Fig. 5. NEP components and photon NEP in dependence on the number of CEBs in a series array. The parameters of CEB: $\mathrm{I}_{\mathrm{JFET}}=5 \mathrm{fA} / \mathrm{Hz}^{1 / 2}, \mathrm{~V}_{\mathrm{JFET}}=3 \mathrm{nV} / \mathrm{Hz}^{1 / 2}, \mathrm{R}=1 \mathrm{kOhm}, \Lambda=0.01 \mu \mathrm{m}^{3}$. The responsivity $S$ is shown for illustration of the effect of the CEB number. Thin lines show asymptotic for S and NEP JFET (Kuzmin 2008a, 2008b).

The optimal number of CEBs in a series array is determined mainly by power load $P_{\mathrm{o}}$ and the volume of absorber $\Lambda$. The general rule of array design is the following: the number of bolometers, $N$, should be increased to split $P_{\mathrm{o}}$ between bolometers up to the point when $P_{0} / N=P_{p h}$, where $P_{p h}=T^{5} p h \Sigma \Lambda$. The phonon power is determined by only one parameter, the volume of the absorber, $\Lambda$. There is no need to increase the number of bolometers more than this figure, because the optical power loading in each bolometer becomes less than the power from phonons. Responsivity is saturated beyond this level.

For a very small absorber volume, the optimal number of bolometers is determined by the interplay between amplifier noise and junction noise. The main rule here is to decrease the 
amplifier noise by increasing the number of CEBs to a level less than that of junction noise realising total noise less photon noise.

\subsubsection{A two-dimensional array of CEBs with distributed dipole antennas and a JFET readout for polarization measurements}

A novel concept of the two-dimensional array of CEBs with focal plane dipole antennas is proposed for sensitive polarization measurements (Kuzmin 2011c). The concept brings a unique combination of perfect polarization resolution due to a large uniform array of CEBs with optimal matching to JFET/CMOS amplifiers due to flexibility in dc connection. Better noise performance is achieved by distribution of power between the number of CEBs and increasing responsivity of bolometers. This arrangement should lead to substantial improvements in sensitivity and dynamic range. Reliability of the 2D array is considerably increased due to parallel/series connections of many CEBs. Polarization resolution should be improved due to uniform covering of a substrate by the 2D array over a large area and absence of beam squeezing to small lumped elements.

The fundamental sensitivity limit of the CEB array is below the photon noise $\mathrm{NEP}=\sqrt{2} \mathrm{hfPo}$, which is referred to as the background-limited performance. Estimations of the CEB noise with JFET readout show an opportunity to realize background-limited performance with NEP less than photon noise $\mathrm{NEP}=510^{-17} \mathrm{~W} / \mathrm{Hz}^{1 / 2}$ at $350 \mathrm{GHz}$ for an optical power load of 5 $\mathrm{pW}$ proposed for BOOMERanG.

The 2D array of CEBs with distributed dipole antennas (Fig. 6) is proposed for receiving one polarization components of the signal in multimode operation and effective matching to a JFET amplifier (Kuzmin 2008c). In this paper we analyse a realization of the CEB array for the $350 \mathrm{THz}$ channel of BOOMERanG. The voltage response is measured by a JFET amplifier in a current-biased mode. The main purpose of the 2D configuration of the array with flexibility for dc connection is to achieve high responsivity (low noise) and to match the total dynamic resistance of the array to the noise impedance of a JFET ( 0.6 MW).

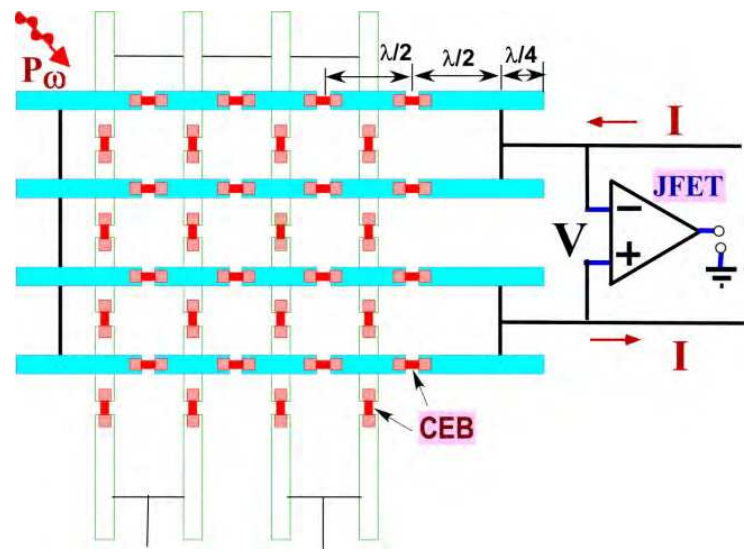

Fig. 6. Single polarization dipole antennas with a 2D array of CEBs $(4 \times 4)$ and a JFET readout. Each dipole antenna will be sensitive only to one polarization component of the RF signal. For illustration an array with a minimum number of $4 \times 4$ CEBs is shown with dc connection of $2 \times 8$ CEBs. Electrical isolation should be done between electrodes of the cross-dipole antennas. 
Probability of failure is considerably decreased due to the parallel combination of several CEBs. For the analysed array we used four CEBs in parallel $(W=4)$ and 16 rows of CEBs in series $(\mathrm{N}=16)$.

In contrast to Day et al. (2004 ), RF connection is made only for series connection in one line. Connection between lines is made only for dc bias by narrow wires.

Further improvement of performance is achieved by placing four bias resistors on chip just near the array of bolometers to decrease the noise of current-bias resistors and to protect scheme from interferences (Kuzmin et al., 1991; Kuzmin \& Haviland, 1991). The RF matching is realized by the resistance of a normal absorber, which is independent of the tunnel junction parameters.

In what follows for analysis of a 2D CEB array we shall use the basic model of the CEB with strong electrothermal feedback due to electron cooling (Kuzmin \& Golubev, 2002; Golubev \& Kuzmin, 2001) and the concept of parallel/series array in current-biased mode (Kuzmin 2008a, 2008b). The operation of a CEB array can be analysed using the heat balance equation for a single $\mathrm{CEB}$, taking into account power distribution between the $\mathrm{NxW}$ bolometers:

$$
2 P_{S I N}\left(V, T_{e}, T_{p h}\right)+\sum \Lambda\left(T_{e}^{5}-T_{p h}^{5}\right)+2 \frac{V^{2}}{R_{j}}+I^{2} R_{a}=\left[P_{0}+\delta P(t)\right] /\left(W^{*} N\right)
$$

Here we assume that the SIN tunnel junctions are current-biased and the voltage is measured by a JFET amplifier. The responsivity $S_{V}$ is described by the voltage response to an incoming power

$$
S_{V}=\frac{\delta V_{\omega}}{\delta P_{\omega}}=\frac{2 \partial V / \partial T}{G_{e-p h}+2 G_{S I N}}
$$

Noise properties are characterized by the noise equivalent power (NEP), which is the sum of three contributions:

$$
N E P_{\text {tot }}^{2}=N * W^{*} N E P_{e-p h}^{2}+N * W^{*} N E P_{S I N}^{2}+N E P_{A M P}^{2}
$$

Here $N E P_{e-p h}$ is the noise associated with electron-phonon interaction (9); $N E P_{S I N}$ is the noise of the SIN tunnel junctions (15). The last term is due to the voltage $d V$ and current $d I$ noise of a JFET, which are expressed in $\mathrm{nV} / \mathrm{Hz}^{1 / 2}$ and $\mathrm{pA} / \mathrm{Hz}^{1 / 2}$ :

$$
N E P_{A M P}^{2}=\left(\delta V^{2}+\left(\delta I^{*}\left(R_{d}+R_{a}\right)^{*} N / W\right)^{2}\right) /\left(S_{V} / W\right)^{2}
$$

The estimations were made for the $350 \mathrm{GHz}$ channel of BOOMERanG.

The optimal way for polarization measurements of two components in the same place is by orthogonal cross-combination of them connected by narrow dc wires for bias and readout connection as shown in Fig. 7. Optical matching could be organised by using Si substrate of resonance length $l / 4$ with backshort behind the substrate (Fig. 7) or by using Si substrate with antireflection coating (Tran \& Page, 2009). An additional Si lens or horn could be added for better concentration of incoming power in the 2D array of CEBs. The main criteria 


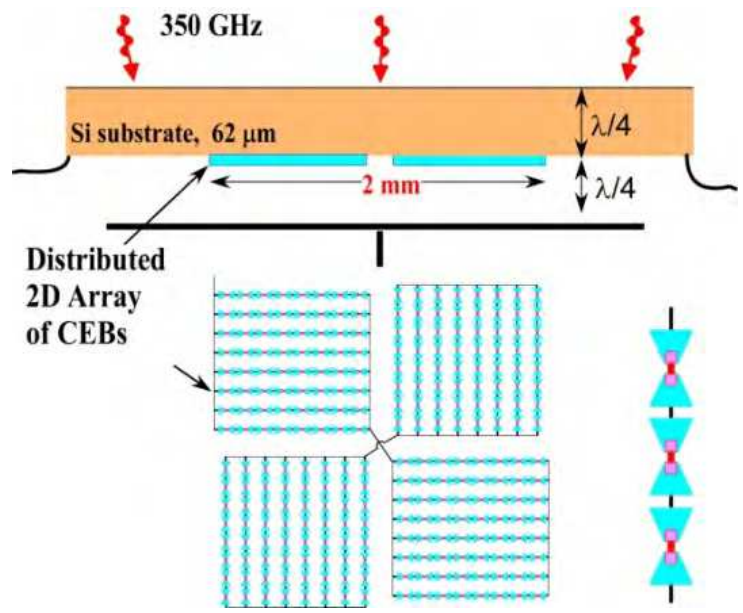

Fig. 7. Focal plane dipole antennas with a 2D array of CEBs consisting of two subarrays of $8 \times 8$ CEBs for each polarization. The RF matching is achieved by using Si substrate of resonance length $l / 4$ with backshort behind the substrate.

are to realise high resolution of polarization at the level of $25 \mathrm{~dB}$ and to keep high efficiency of matching. The results of the simulation for the $2 \mathrm{D}$ array consisting of two sections of $8 \times 8$ CEBs for each polarization for different combination of dc connections are shown in Fig. 8 . Fig. 8 shows that for all analysed dc combinations of width $(\mathrm{W})$ and length $(\mathrm{N})$ of the array we realize a total NEP of bolometer less than photon noise NEPphot. Fig. 8 shows that the optimal realization is for an array of $4 \times 32$. Degradation of performance for a wider array of $8 \times 16$ CEBs is related to a decrease of responsivity $S=d V / d P$.

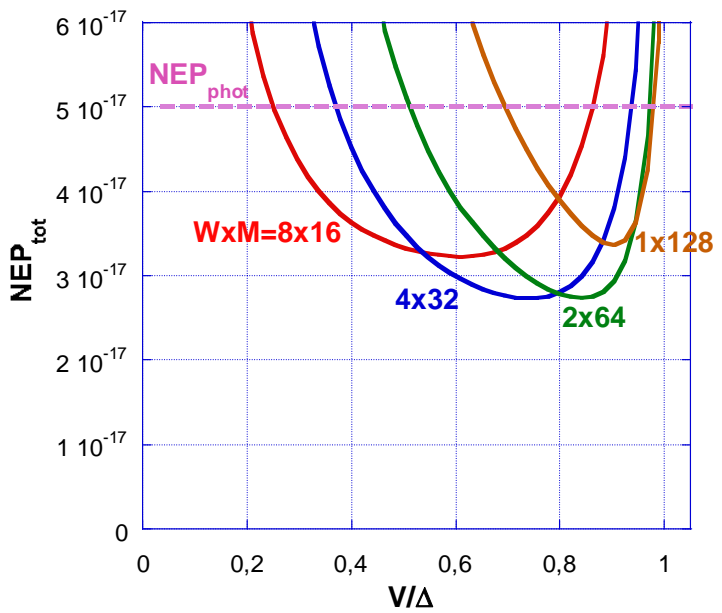

Fig. 8. NEP components of the 2D array of CEBs at $350 \mathrm{GHz}$, comprising two subarrays of 8x8 CEBs for each polarization (Fig. 4). Different combinations of CEBs (WxN) for dc connection are simulated for matching with the JFET readout. Power load $=5 \mathrm{pW}, \mathrm{I}_{\mathrm{JFET}}=5$ $\mathrm{fA} / \mathrm{Hz}^{1 / 2}, \mathrm{~V}_{\mathrm{JFET}}=3 \mathrm{nV} / \mathrm{Hz}^{1 / 2}, \mathrm{R}=3 \mathrm{kOhm}, L=0.01 \mathrm{~mm}^{3}, \mathrm{~T}=300 \mathrm{mK}$. 
Degradation of performance for more narrow arrays of $2 \times 64$ and $1 \times 128$ CEBs is related to an increase of total dynamic resistance of the array more than the noise impedance of a JFET amplifier. To compensate this mismatch, the optimal operation point is shifted to higher voltages with lower resistance. From these simulations the optimal array in the sense of noise and reliability is a $2 \mathrm{D}$ array with four CEBs connected in parallel and 32 CEBs connected in series.

The main progress in matching with the JFET readout is achieved by proper selection of the parallel and series combination of CEBs in 2D arrays, and selection of proper resistance of SIN junctions (Fig. 8). Results of simulation for the 2D array with optimal combination of $4 \times 32$ CEBs for various resistances of the SIN tunnel junction are shown in Fig. 9.

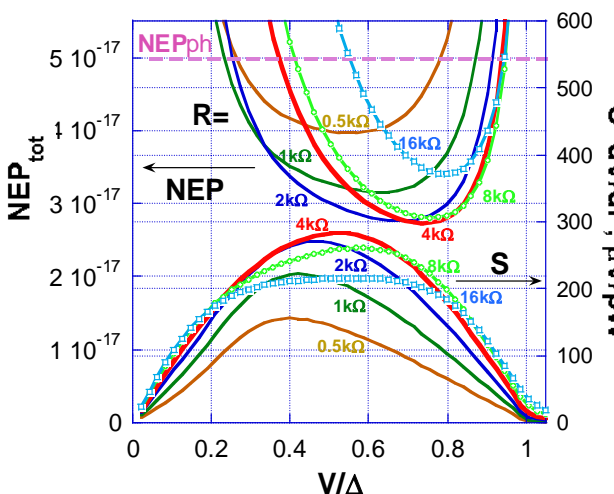

(a)

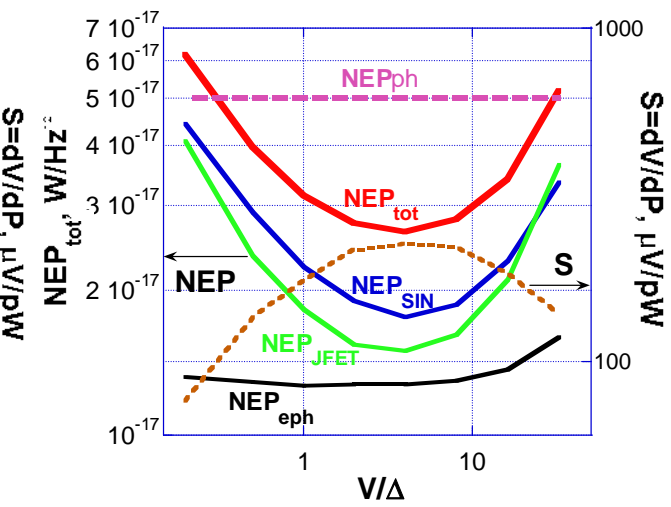

(b)

Fig. 9. (a) Voltage dependence of total NEP and responsivity $S=d V / d P$ for the $2 \mathrm{D}$ array of CEBs, comprising two subarrays of $8 \times 8$ CEBs for one polarization with optimal combination of $4 \times 32$ CEBs for dc connection for different values of SIN junction resistance R;

(b) Dependence of NEP components of the same 2D array on resistance of SIN tunnel junction. The parameters of CEBs are the same as in Fig. 8.

Optimal value of resistance for minimum NEP is $3 \mathrm{kOhm}$. Noise components in dependence on resistance are shown in Fig. 9b. The total noise is determined mainly by noise of SIN tunnel junction (15) and noise of amplifier (JFET) (21). The electron-phonon noise (9) is small due to the small volume of the absorber and low temperature independently on a rather large number of CEBs.

Finally, a novel concept of the 2D array of CEBs with distributed dipole Antennas has been proposed for polarization measurements with high resolution of polarization components. This concept provides more flexibility in matching to JFET readout and better noise performance due to the distribution of power between the number of junctions with decreasing power load and the increasing responsivity of each individual CEB. Polarization resolution should be improved due to the absence of beam squeezing to small lumped elements. The reliability of 2D array is considerably increased due to the parallel/series connections of many CEBs. 


\subsubsection{D array of CEBs with distributed cross-dipole antennas for multimode measurements of both polarization components}

A novel concept of the 2D array of CEBs with a cross-dipole antenna is proposed for ultrasensitive multimode measurements of both polarization components of an RF signal (Kuzmin 2011a). This concept gives the opportunity of avoiding complicated combinations of two schemes to measure simultaneously both polarization components. The optimal concept of the CEB including a superconductor-insulator-normal (SIN) tunnel junction and an Andreev SN contact is used for better performance. This concept provides better matching with the JFET readout, suppresses charging noise related with Coulomb blockade due to the small area of tunnel junctions and decreases the volume of a normal absorber for further improvement of noise performance. The reliability of $2 \mathrm{D}$ array is considerably increased due to the parallel/series connections of many CEBs.

Estimations of the CEB noise with the JFET readout show an opportunity to realize NEP less than photon noise $\mathrm{NEP}=410^{-19} \mathrm{~W} / \mathrm{Hz}^{1 / 2}$ at $7 \mathrm{THz}$ for an optical power load of $0.02 \mathrm{fW}$.

The CEB is a planar antenna-coupled superconducting detector that can easily be matched with any planar antenna. Promising direction is distributed focal plane antennas (Kuzmin, 2008c; Day et al., 2004). These antennas could help to avoid horns or Si lenses for matching with bolometers and could be used in a multimode regime for wide band spectrometers. To achieve RF matching to a distributed focal plane antenna, a series array of the CEB and JFET readouts have been analysed (Kuzmin, 2008c). However, this configuration has several disadvantages for the spectrometer: the antenna measures only one polarization component, resistance is too high for matching with the JFET and the Coulomb blockade starts to become important at low temperatures due to small junction capacitance, and the probability of failure is increased proportionally to the number of bolometers in the series array. To avoid these problems, a novel concept of the 2D array of CEBs with a distributed

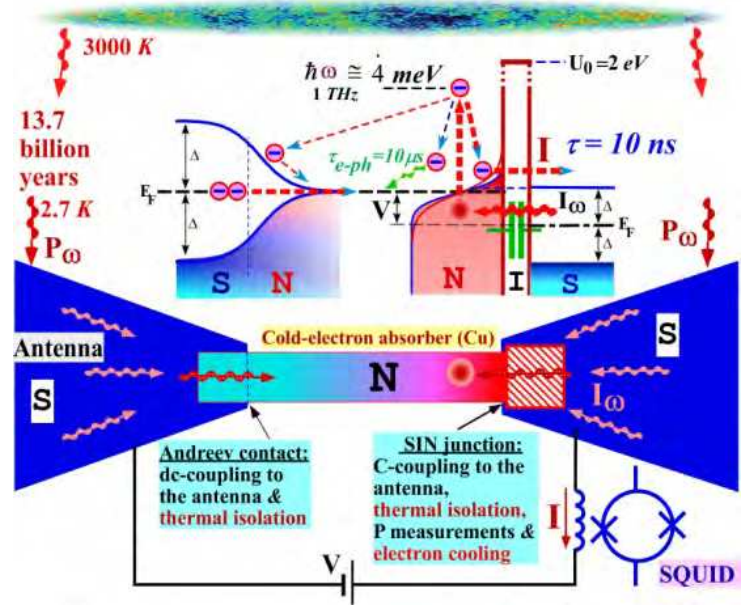

Fig. 10. Schematic of the optimal CEB with capacitive coupling to the antenna (Kuzmin, 2006). A normal nanoabsorber is coupled to the antenna through the capacitance of a SIN tunnel junction and through a SN Andreev contact. The SIN tunnel junction is used also for thermal isolation, electron cooling and for reading out the signal with a SQUID or a JFET. 
cross-dipole antenna is proposed. The optimal CEBs with a SIN tunnel junction and Andreev contact (Kuzmin, 2006) is used to overcome the above-mentioned problems (Fig. 10). The system is proposed for SPICA and MILLIMETRON spectrometers, and other ultrasensitive cosmology instruments.

Detection using CEB is obtained by allowing the incoming signal to pass from the antenna to the absorber through the capacitance of a SIN tunnel junction and through a SN Andreev contact. Using this optimal concept of CEB, we achieve several advantages: resistance is decreased for better matching with the JFET, the Coulomb blockade is suppressed due to the absence of the second junction creating SET transistor (Kuzmin \& Likharev, 1987; Kuzmin \& Safronov, 1988) and the effective volume of the absorber is decreased due to the proximity effect of the Andreev contact.

The main mode of CEB operation is a concept employing a 2D array of CEBs (Fig. 11) for effective matching to a JFET amplifier (Kuzmin, 2011). A distributed cross-dipole antenna (Fig. 11) is proposed for receiving both components of the signal in multimode operation. In this paper we analyse a realization of the CEB array for the $7 \mathrm{THz}$ channel.

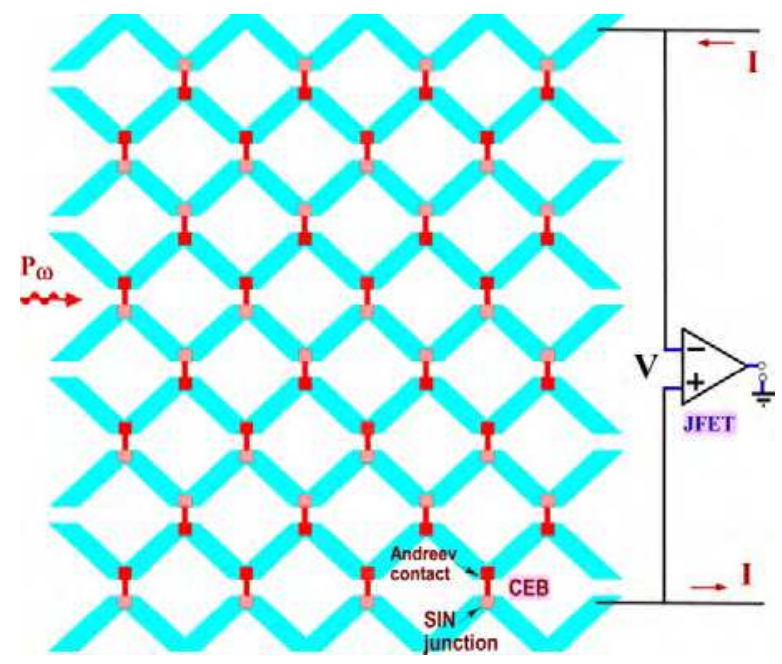

Fig. 11. Distributed cross-dipole antenna with a 2D array of CEBs and a JFET readout. This double polarization antenna is sensitive to both components of the RF signal.

The voltage response is measured by a JFET amplifier in a current-biased mode. The main purpose of the $2 \mathrm{D}$ array for readout is to match the total dynamic resistance of the array to the noise impedance of a JFET $(\sim 0.6 \mathrm{M} \Omega)$.

Probability of failure is considerably decreased due to the parallel combination of several CEBs. For the analysed array we used four CEBs in parallel $(\mathrm{W}=4)$ and eight rows of CEBs in series $(\mathrm{N}=8)$.

Further improvement of performance is achieved by placing four bias resistors on chip for decreasing noise of bias resistors and interferences (Kuzmin, 1991). RF matching is realised by the resistance of a normal absorber. 
In the following analysis of a 2D CEB array we shall use the basic model of the CEB with strong electrothermal feedback due to electron cooling (Kuzmin \& Golubev, 2002; Golubev \& Kuzmin, 2001) and the concept of a parallel/series array in current-biased mode (Kuzmin 2008a, 2008b) similar to section 2.2.2. The operation of a CEB array can be analysed using the heat balance equation for a single $\mathrm{CEB}$, taking into account power distribution between the NxW bolometers.

The estimations were made for the $7 \mathrm{THz}$ channel of SPICA. Results of the simulation for the $2 \mathrm{D}$ array with the JFET readout are shown in Fig. 12. The Fig. 12 shows considerable improvement of noise properties for transition from the CEB with a double SIN junction to the CEB with a single SIN junction and an SN Andreev contact.

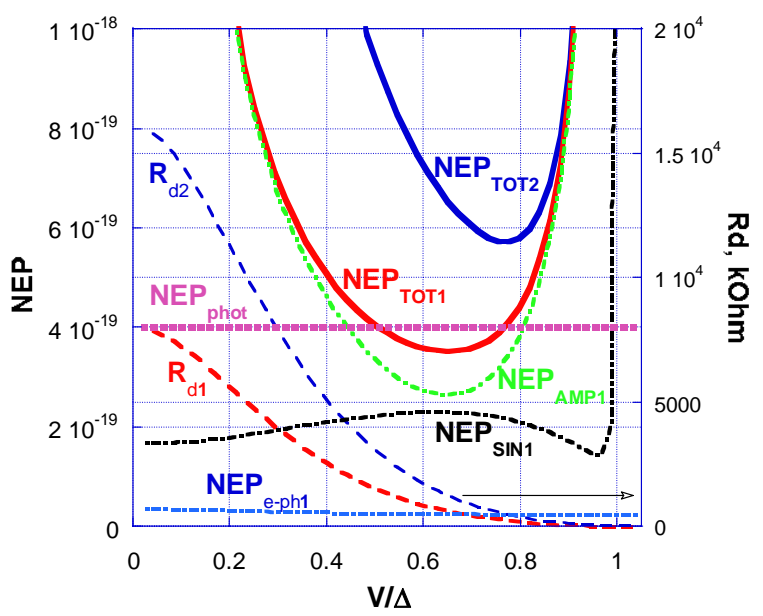

Fig. 12. NEP components for the CEB with a single SIN junction and an SN Andreev contact ( " 1 ") and for the CEB with a double SIN junction ( "2"). Dynamic resistance, $\mathrm{Rd}$, is shown for both cases referred to on the right axis. Parameters: $\mathrm{I}_{\mathrm{amp}}=5 \mathrm{fA} / \mathrm{Hz}^{1 / 2}, \mathrm{~V}_{\mathrm{amp}}=3 \mathrm{nV} / \mathrm{Hz}^{1 / 2}$ (JFET), $\mathrm{R}=4 \mathrm{kOhm}$, Volume $\Lambda=0.002 \mathrm{um}^{3}, \mathrm{~T}=70 \mathrm{mK}, \mathrm{Tc}=400 \mathrm{mK}$. The $2 \mathrm{D}$ array consists of four CEBs in parallel $(\mathrm{W}=4)$ and eight CEBs in series $(\mathrm{N}=8)$.

Improvement of NEP for single SIN junction is achieved due to the double decrease of dynamic resistance, $\mathrm{Rd}$, and proportional decrease of the amplifier noise determined by a product of the current noise of the amplifier and the $\mathrm{Rd}(20)$. The total noise is determined mainly by the noise of the JFET amplifier (21) and the noise of the SIN tunnel junction (15).

The electron-phonon noise (9) is small due to the small volume of the absorber and low temperature. As we can see from Fig. 12, noise performance for the optical power load of $0.02 \mathrm{fW}$ fits requirements with NEPtot<NEPphot for $\mathrm{R}=4 \mathrm{kOhm}$.

The simulations show that better NEP can be obtained with a decreased gap of Al to the level of $400 \mathrm{mK}$. This suppression of the gap can be obtained with additional evaporation of any normal metal $(\mathrm{Cu}, \mathrm{Ti}, \ldots)$ on the top of the counter electrode.

The results of the simulation for the 2D array for different resistances of the SIN tunnel junction are shown in Fig. 13. As we can see from Fig. 13, noise performance for the optical power load of $0.02 \mathrm{fW}$ fits requirements of SAFARI with NEPtot<NEPphot for R $>4 \mathrm{kOhm}$. 


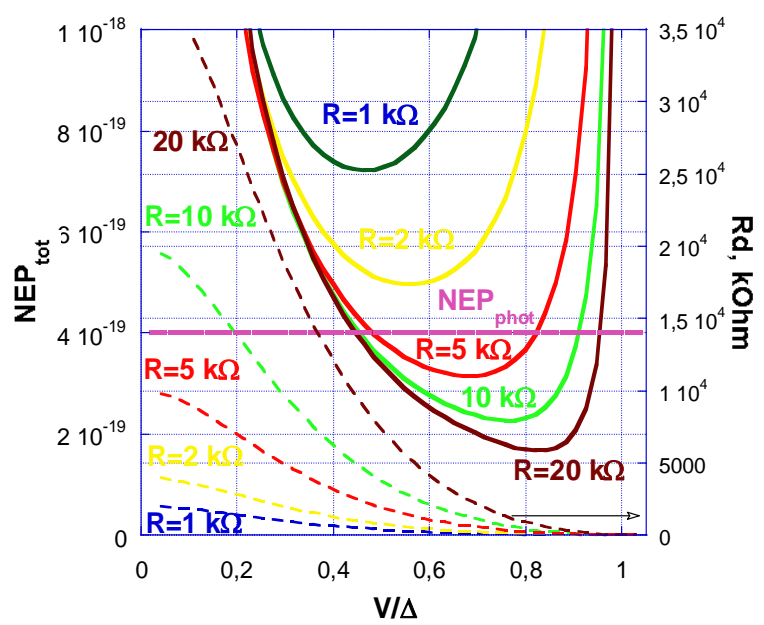

Fig. 13. NEP components of the 2D array of CEBs with the JFET readout at $7 \mathrm{THz}$ with power load of $0.02 \mathrm{fW}$ for $\mathrm{I}_{\mathrm{JEET}}=5 \mathrm{fA} / \mathrm{Hz}^{1 / 2}, \mathrm{~V}_{\mathrm{JFET}}=3 \mathrm{nV} / \mathrm{Hz}^{1 / 2}, \Lambda=0.002 \mu \mathrm{m}^{3}, \mathrm{~T}=70 \mathrm{mK}$, $\mathrm{Tc}=400 \mathrm{mK}$ for different values of normal resistance $\mathrm{R}$.

Improvement of NEP for larger $R$ is achieved due to the increase of responsivity $S v=d V / d P$ proportional to R. For the left slope of NEP curves, this improvement is compensated by the increase of amplifier noise due to the product of the current noise of the amplifier and dynamic resistance of the junction. For the right slope of NEP curves, we do not have this limitation due to the noise of the amplifier and improvement is due to a slight decrease of overheating related with dissipation of power in a leakage resistance due to electron cooling.

The main progress in matching with the JFET readout is achieved by proper selection of the parallel and series combination of CEBs and selection of proper resistance of SIN junctions (Fig. 13). Some progress in NEP is realised due to replacing two SIN junctions by one SIN and one SN Andreev contact (Fig. 12). Some progress in better performance is achieved also due to proper suppression of Delta of the top superconducting electrode to the level of 400 $\mathrm{mK}$ (instead of $1.2 \mathrm{~K}$ for clean $\mathrm{Al}$ ). The internal overheating of CEBs by applied voltage is decreased and we can realise arrays of any size (even with $N>100$ ).

\subsection{CEB in voltage-biased mode}

\subsubsection{SCEB with SIS' tunnel junctions and Josephson junction and a SQUID readout}

A novel concept of a superconducting cold-electron bolometer (SCEB) with a superconductor-insulator-weak superconductor (SIS') tunnel junction and Josephson junction has been proposed (Fig. 14) (Kuzmin, 2008d). The main innovation of this concept is utilising the Josephson junction for dc and HF contacts, and for thermal isolation. The SIS junction is used also for electron cooling and dc readout of the signal. The SIS junction is designed in loop geometry for suppression of the critical current by a weak magnetic field. The key to this concept is that the critical current of the Josephson junction is not suppressed by this weak magnetic field and can be used for dc contact. Due to this innovation, a robust two layer technology can be used for fabrication of reliable structures. A direct connection of SCEBs to a four-probe antenna has been proposed for effective RF coupling. 


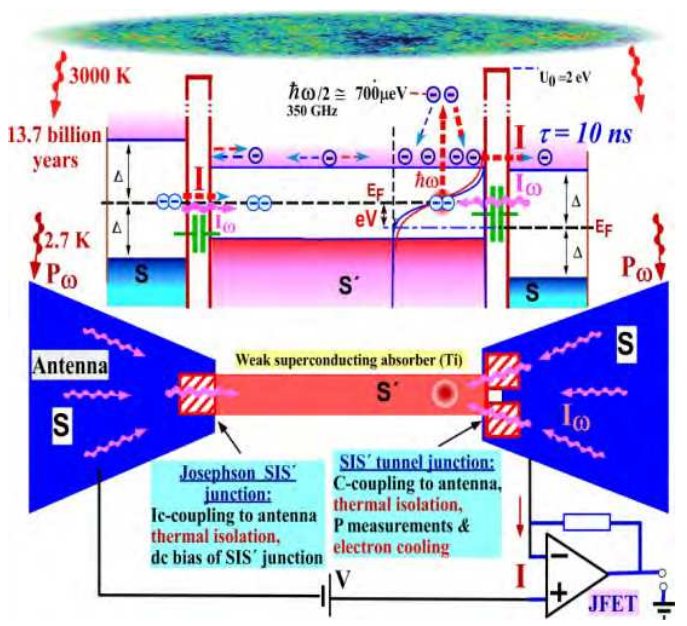

Fig. 14. Schematic of a SCEB with SIS ' and Josephson tunnel junctions and a JFET readout. The SIS' junction is used for capacitive coupling to the antenna, thermal isolation, electron cooling and dc readout by a JFET. The Josephson junction is used for dc and HF contacts, and for thermal isolation.

The main innovation of the SCEB in comparison with the CEB with double SIN junction (Kuzmin, 2000, 2001, 2004; Kuzmin \& Golubev, 2002) and CEB with SIN and Andreev contact (Kuzmin, 2006) is effective utilization of the Josephson junction for dc and HF contacts, and for thermal isolation. The SIS junction (for HF coupling, thermal isolation, electron cooling and dc readout) is proposed in loop geometry for suppression of the critical current by a weak magnetic field. Strikingly, the critical current of Josephson junction is not suppressed by this weak magnetic field. As a result of this innovation, a robust two layer technology can be used for fabrication of both SIS' and Josephson tunnel junctions. In this paper we analyse realization of the SCEB for $350 \mathrm{GHz}$ channel of BOOMERanG and other cosmology instruments.

Noise properties are characterized by the NEP

$$
N E P_{\text {total }}^{2}=N E P_{e-p h}^{2}+N E P_{S I S^{-}}^{2}+\frac{\delta I^{2}}{S_{I}^{2}} .
$$

Here NEP2e-ph is the noise due to electron-phonon interaction; NEP2SIS' is the noise of an SIS' junction and $\delta I^{2} / S^{2} I$ is the noise of a JFET amplifier.

The noise of the SIS' junction has three components: shot noise 2eI/S2I, the heat flow noise and the correlation term between these two processes (Golwala, S. et al., 1997; Golubev \& Kuzmin, 2001)

$$
N E P_{S I S^{\prime}}^{2}=\delta P_{\omega}^{2}-2 \frac{\delta P_{\omega} \delta I_{\omega}}{S_{I}}+\frac{\delta I_{\omega}^{2}}{S_{I}^{2}}
$$

This correlation is a form of the electrothermal feedback discussed earlier (Mather, 1982). For a superconductor absorber with concentration of electrons just near the gap, this 
anticorrelation is very strong and could lead, in first approximation, to almost $100 \%$ compensation of the shot noise:

$$
\delta P_{\omega}^{2}=2 \Delta P_{0}, \delta I_{\omega}^{2}=2 e^{2} P_{0} / \Delta, S_{I}=e / \Delta, N E P_{S I S^{\prime}}^{2} \cong 0
$$

For every chosen voltage we first solve the heat balance equation, find the electron temperature in the absorber, taking into account the effect of the electron cooling, and then determine current responsivity and NEP.

For optical power load of $\mathrm{P}_{0}=5 \mathrm{pW}$ for each polarization of $350 \mathrm{GHz}$ channel the photon noise is $N E P_{\text {phot }}=\sqrt{2 P_{0}{ }^{*} h f}=4.3 * 10^{-17} \mathrm{~W} / \mathrm{Hz}{ }^{1 / 2}$.

Figure 15 shows a simulation of the different contributions to the total NEP of the detector for an optimised geometry of the bolometer. We see that for a range of bias voltage from 170 $\mu \mathrm{V}$ to $210 \mu \mathrm{V}$, the total NEP of the SCEB is well below the photon noise: $\mathrm{NEP}_{\text {tot }}<\mathrm{NEP}_{\text {phot }}$. The range of voltages less than $170 \mu \mathrm{V}$ is not recommended for use because, due to negative slope of the IV-curve, the operation point would be unstable. In addition, the $\mathrm{NEP}_{\text {tot }}$ of the SCEB is dominated by shot/heat noise of the detector (NEPSIS) corresponding to a background limited mode of operation.

Figure 15b illustrates the effect of the noise reduction of SIS'tunnel junction. The figure shows all components of SIS'noise: $\mathrm{NEP}_{\text {shot }} \mathrm{NEP}_{\text {heat }}$ and correlation term $(\mathrm{dPdI})^{1 / 2}$. The final noise, $\mathrm{NEP}_{\mathrm{SIS}}$, is clearly less than original noise components. The effect is stronger than for SIN junction noise (Golubev \& Kuzmin, 2001) due to the well-defined level of quasiparticle energy just near the gap.

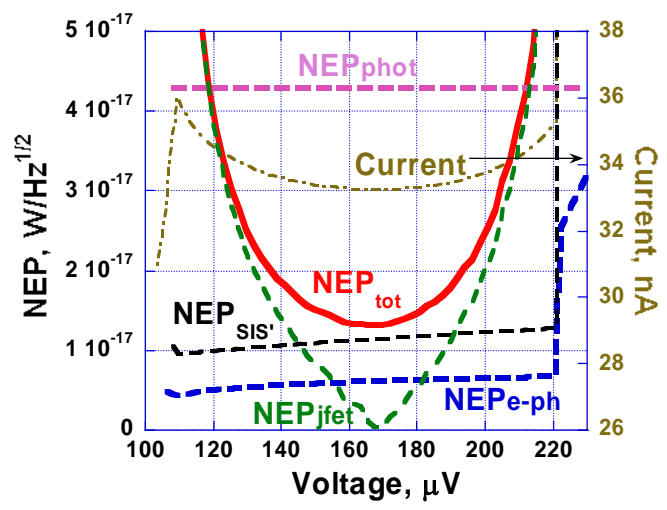

(a)

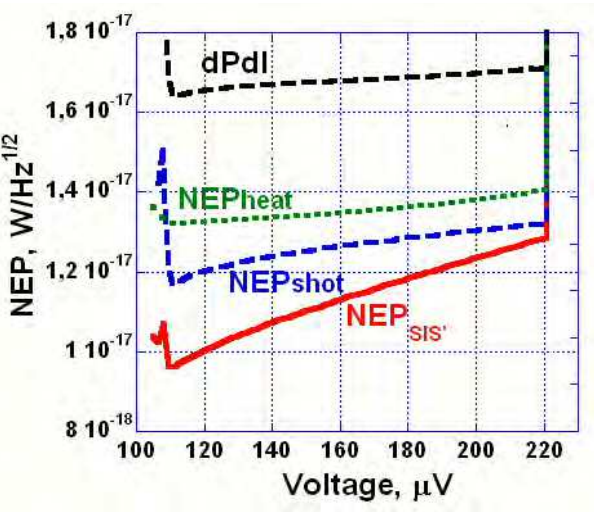

(b)

Fig. 15. (a) NEP components of the SCEB for $\mathrm{I}_{\mathrm{JFET}}=5 \mathrm{fA} / \mathrm{Hz}^{1 / 2}, \mathrm{~V}_{\mathrm{JFET}}=3 \mathrm{nV} / \mathrm{Hz}^{1 / 2}, \mathrm{R}=0.2 \mathrm{kOhm}$, $\Lambda=0.04 \mathrm{um}^{3}$. The $\mathrm{NEP}_{\text {tot }}$ is less than $\mathrm{NEP}_{\text {phot; }}$ (b) Resulting SIS junction noise with strong cancellation of the $\mathrm{NEP}_{\text {shot }}$ and $\mathrm{NEP}_{\text {heat }}$ due to the anticorrelation term $\mathrm{dPdI}$ between them.

\subsubsection{Parallel array of CEBs with distributed slot antennas and a SQUID readout}

An innovative concept of the parallel array of CEBs with superconductor-insulator-normal (SIN) tunnel junctions has been proposed for distributed focal plane slot antennas (Kuzmin, 
2008c). The parallel connection of CEBs with SIN tunnel junctions in voltage-biased mode is optimal for a slot antenna (Fig. 16). Some improvement of properties can be achieved by using optimal configuration of CEB with SIN junction and SN Andreev contact shown in Fig. 7 (Kuzmin, 2006). Any use of a double junction in voltage-biased mode (Kuzmin, 2001,2004; Kuzmin \& Golubev, 2002) would lead to splitting power between two junctions and some degradation of responsivity.

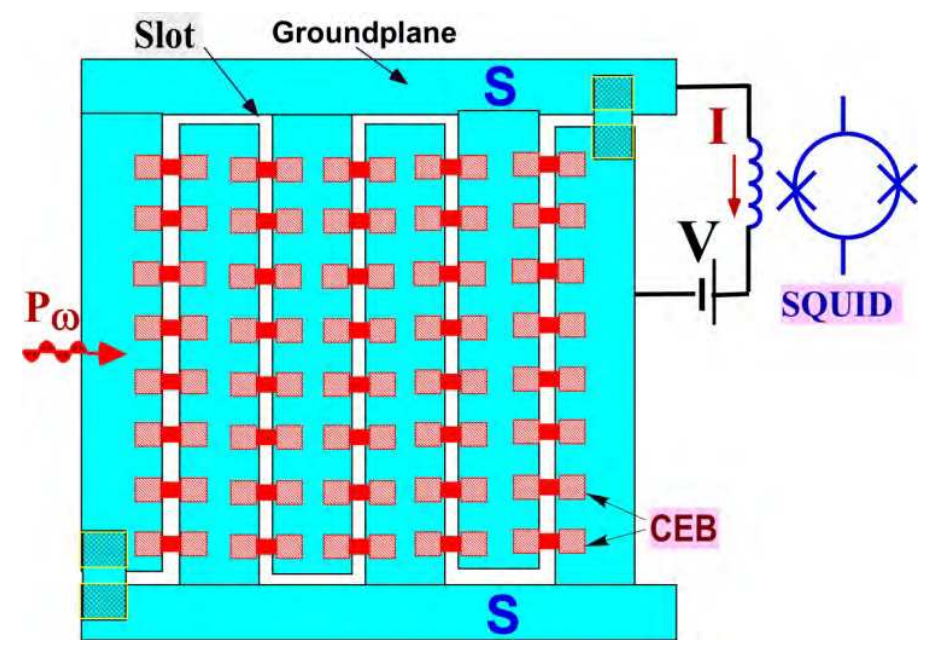

Fig. 16. A distributed single polarization slot antenna (Day, 2004) with a parallel array of CEBs (Kuzmin, 2008c) and a SQUID readout. This slot antenna will be sensitive only to horizontal components of the RF signal. The CEBs with SIN tunnel junctions and Al-Al SQUID could be fabricated on the same chip in one vacuum cycle.

An important feature of the design is that the volume of the normal metal is partly squeezed due to the proximity effect of the superconducting electrode of the Andreev contact. This squeezing further increases the efficiency of the electron cooling without degrading the HF coupling. Estimations of the CEB noise with the SQUID readout have shown an opportunity to realize background-limited performance for a typical power load of $5 \mathrm{pW}$ proposed for BOOMERanG.

The Al-Al SQUID could be fabricated on the same chip as CEB with SIN tunnel junctions in the same vacuum circle. Simultaneous fabrication of CEB and SQUID on-chip would create more reliable structures and avoid interferences due to wire interconnections of the systems.

We have analysed the concept of an optimal CEB for $70 \mathrm{GHz}$ channel of B-Pol polarometer in the presence of the typical power load $\left(P_{0}=0.2 \mathrm{pW}\right)$.

Fig. 17 shows the results of a simulation of a CEB with a single SIN junction, with realistic parameters for the tunnel junction and absorber, and values of SQUID noise from 0.1 $\mathrm{pA} / \mathrm{Hz}^{1 / 2}$ to $0.8 \mathrm{pA} / \mathrm{Hz}^{1 / 2}$. The level of NEPphot has been achieved for SQUID noise lower than $0.5 \mathrm{pA} / \mathrm{Hz}^{1 / 2}$.

Optimal number of CEBs in series array. The optimal number (Fig. 18) is determined mainly by the power load Po and the volume of absorber $\Lambda$. The general rule of array design is the 


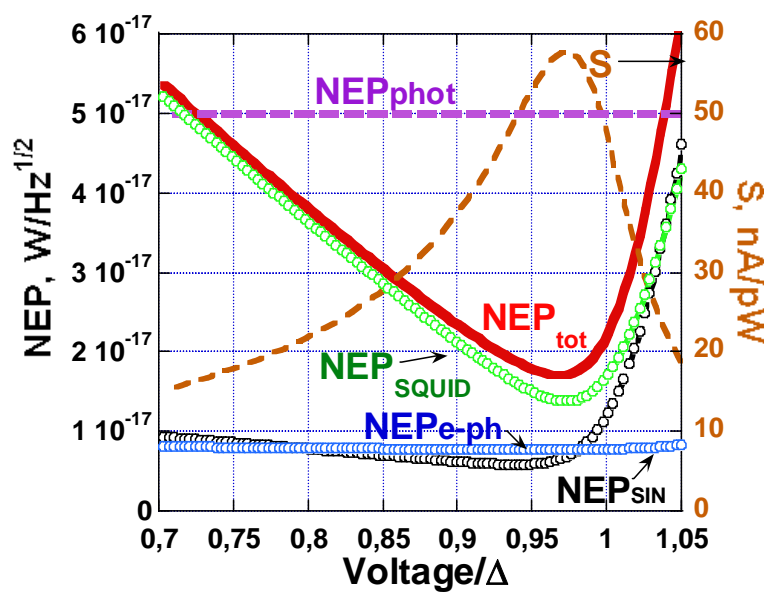

Fig. 17. Total NEP of the 40 CEB array with SIN tunnel junctions for the $350 \mathrm{GHz}$ channel, with a SQUID noise current of $0.8 \mathrm{pA} / \mathrm{Hz}^{1 / 2}$. Parameters: $\mathrm{R}=0.2 \mathrm{kOhm}, \mathrm{S}=1 \mu \mathrm{m}^{2}, \mathrm{Vol}=0.005 \mathrm{um}^{3}$, power load $P_{0}=5 \mathrm{pW}, \mathrm{T}=300 \mathrm{mK}$. The NEPphot $=5^{\star} 10^{-17} \mathrm{~W} / \mathrm{Hz}^{1 / 2}$ is shown by dashed line.per polarization component. For the $70 \mathrm{GHz}$ channel, NEPphot $=4.3^{\star} 10^{-18} \mathrm{~W} / \mathrm{Hz}^{1 / 2}$.

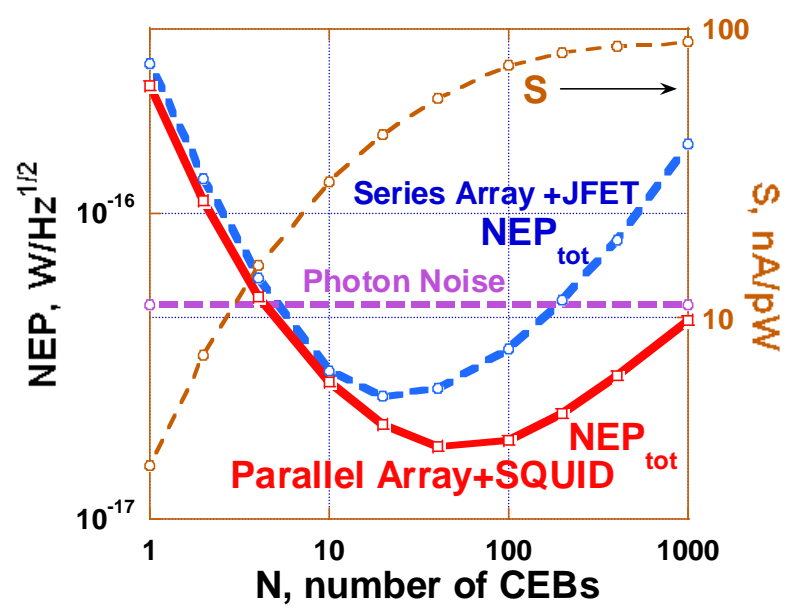

Fig. 18. NEP components and photon NEP in dependence on the number of CEBs in a voltage-biased parallel array with SQUID and in current-biased series array with JFET (section 2.2.2, $M=1$ ). The parameters of CEBs are the same as in Fig. 17. The responsivity $S$ is shown for a parallel array for illustration of the effect of the CEB number.

following: the number of bolometers, $\mathrm{N}$, should be increased to split Po between bolometers up to the point when $P_{0} / N=P_{p h}$, where $P_{p h}=T^{5}{ }_{p h} \Sigma \Lambda$. The phonon power is determined by only one parameter, the volume of the absorber, $\Lambda$. There is no need to increase the number of bolometers more than this figure because the optical power loading in each bolometer becomes less than the power from phonons. Responsivity is saturated after this level. 


\section{Experimental realization of the Cold-Electron Bolometer (CEB)}

\subsection{Technology of fabrication}

The first CEB samples were fabricated using the Dolan shadow evaporation technique with a suspended mask made by e-beam lithography (Dolan, 1977). The technique is widespread for fabrication of sub-micron junctions for a wide range of applications. The technique utilizes a suspended mask fabricated using two-layer resist and shadow angle evaporation of two layers of metal. In the first CEB samples the aluminium electrode was thermally evaporated at an angle of $55^{\circ}$ relative to the surface normal up to a thickness of about $60 \mathrm{~nm}$ (Kuzmin et al., 2002, Tarasov et al., 2002; Agulo et al., 2005). The tunnel barrier was formed by oxidizing the electrode for two minutes at a pressure of $5 \times 10^{-2} \mathrm{mbar}$. The normal metal absorber was created by evaporating $30 \mathrm{~nm}$ of chromium and then $30 \mathrm{~nm}$ of copper at an angle of $0^{\circ}$. Cr was used to improve the impedance matching of the antennas to the normal metal and also for better adhesion of $\mathrm{Cu}$ to the substrate.

The first problem of this technology was related to evaporation of the normal metal as a top layer thicker than the base layer of Al. It led to rather high thickness of the absorber that degraded the performance of the bolometer. We tried an alternative technique, a so-called direct write technology with first depositing the normal metal absorber, its etching and oxidation and deposition of the superconducting $\mathrm{Al}$ layer after the second e-beam lithography (Otto et al., 2010). The disadvantage of this method is degradation of tunnel barrier properties during the second lithography process. To avoid this problem we modified the shadow evaporation technique by first depositing a bilayer of $\mathrm{Cr} / \mathrm{Al}$ normal metal, oxidizing it, and then depositing the second superconducting Al layer (Tarasov et al., 2009).

The second serious problem was related to the strong limitation for an area of tunnel junctions in the Dolan shadow evaporation technique by submicron size that created serious problems with the capacitive coupling of the CEB and strength of electron cooling. The breakthrough in this problem occurred after the invention of the Advanced Shadow Evaporation Technique (ASHET) for large area tunnel junctions (Kuzmin, 2011b). This technique is dual to Dolan technique and gives the opportunity for fabrication of large area tunnel junctions (with area $>>1 \mu \mathrm{m}^{2}$, really unlimited) in combination with nanoscale size absorbers.

\subsection{Electron cooling experiments}

The works on electron cooling are devoted to development of a CEB with capacitive coupling by SIN tunnel junctions to the antenna for sensitive detection in the $\mathrm{THz}$ region (Kuzmin et al., 2004; Agulo, I., et al., 2005). We used 4-junction geometry with Al-AlOx$\mathrm{Cr} / \mathrm{Cu}$ tunnel junctions and Au traps (Fig. 19).

The maximum decrease in electron temperature of about $200 \mathrm{mK}$ has been observed at bath temperatures of 300-350 mK. Effective electron cooling was realized due to the improved geometry of the cooling tunnel junctions (quadrant shape of the superconducting electrode) and effective $\mathrm{Au}$ traps just near the junctions $(\approx 0.5 \mu \mathrm{m})$ to decrease reabsorption of quasiparticles after removing them from normal metal.

Figure 20 shows the electron cooling curves and the theoretical fit for different temperatures for the structure with normal metal traps. In Fig. 20 we used for experimental curves both the experimental calibration curve of measured $V\left(T_{p h}\right)$ and theoretical estimation of $V\left(T_{e}\right)$ shown in the same figure. The best coincidence of theoretical fit was obtained using the $\mathrm{T}^{6}$ - 
temperature dependence. The experimental results show very good agreement with the theory for phonon temperatures in the range from $300 \mathrm{mK}$ to $400 \mathrm{mK}$ (Fig. 20).

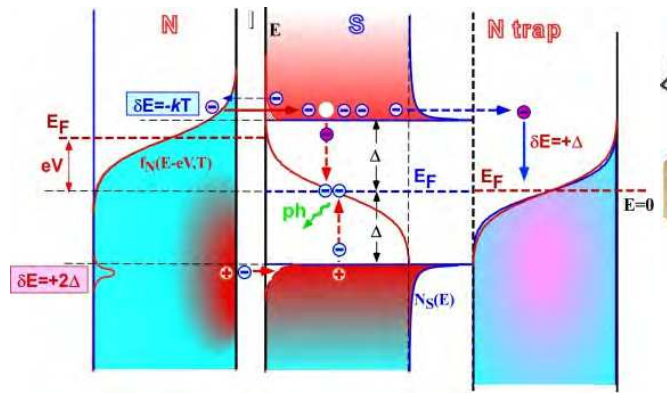

(a)

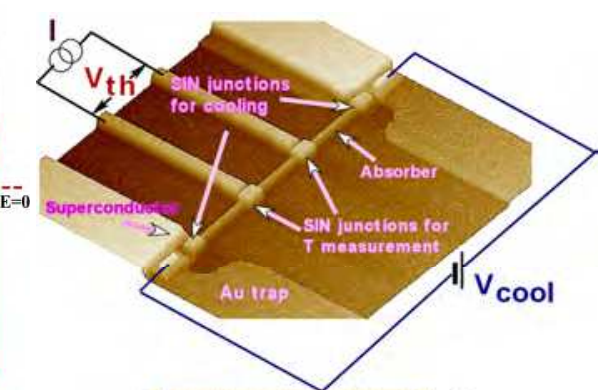

(b)

Fig. 19. (a) Energy diagram illustrates the principle of the electron cooling and the problem of reabsorption of the phonons after recombination of the quasiparticles. The normal metal trap is introduced to avoid this reabsorption. (b) The AFM image of the cooling structure was made using the shadow evaporation technique. The Au trap was evaporated prior to the evaporation of the $\mathrm{Al}-\mathrm{AlOx}-\mathrm{Cr} / \mathrm{Cu}$ tunnel junctions.

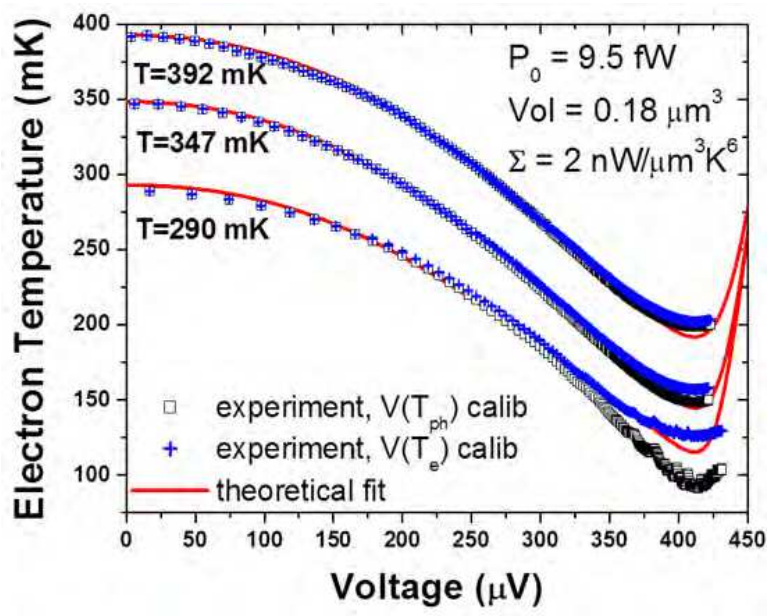

Fig. 20. Electron temperature measured for phonon temperatures of $392 \mathrm{mK}, 347 \mathrm{mK}$ and $290 \mathrm{mK}$. Theoretical fit for background power of $9.5 \mathrm{fW}$, absorber volume $0.18 \mu \mathrm{m}^{3}$.

\subsection{DC qualification of the CEB}

The electrical NEP measurements are realized in a special test structure with two junctions for electron heating and two junctions for temperature sensing (Agulo et al., 2005). In this case we can apply some small dc or ac heating power and measure the voltage response to such power $\mathrm{dV} / \mathrm{dP}$. The bolometer responsivity and the NEP were measured by applying a modulated heating current through the absorber. The frequency of modulation varied from $35 \mathrm{~Hz}$ to $2 \mathrm{kHz}$. The best responsivity of $15 \times 10^{9} \mathrm{~V} / \mathrm{W}$ was obtained at $35 \mathrm{~Hz}$. NEP of better than $2^{*} 10^{-18} \mathrm{~W} / \mathrm{Hz}^{1 / 2}$ 
was measured for modulation frequencies above $100 \mathrm{~Hz}$. The bolometer time constant below 5 $\mu$ s was estimated using the experimental device parameters (Agulo et al., 2005).
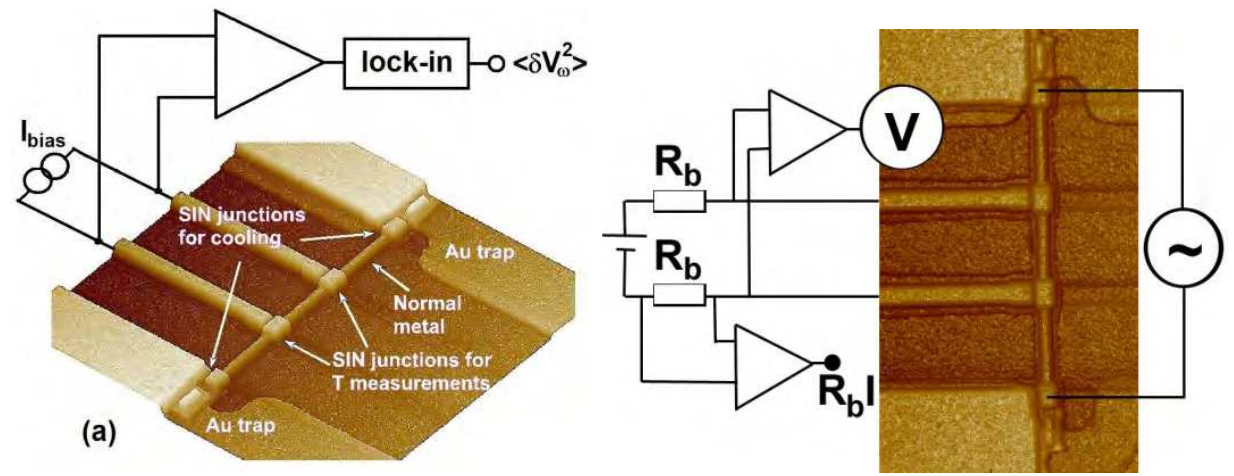

Fig. 21. Schematic diagram of the noise (left image) and responsivity (right image) measurements. The responsivity was measured by applying a heating power through the outer junctions with dc current and with modulated current, and then measuring the voltage response. The total noise was measured using a lock-in amplifier.

\subsection{Quasioptical measurements}

The responsivity of the parallel/series array of CEBs with a cross-slot antenna was measured using a blackbody radiation source equipped with thermometer and resistive heater (Tarasov et al., 2009; Tarasov et al., 2011). The blackbody radiation source (Fig. 23) was placed in front of the CEB attached to an extended hemisphere sapphire lens with antireflection coating. The source was mounted on a $3 \mathrm{~K}$ temperature stage of $\mathrm{He} 3$ cryostat. The temperature of blackbody can be varied in the range of 3-20 K.

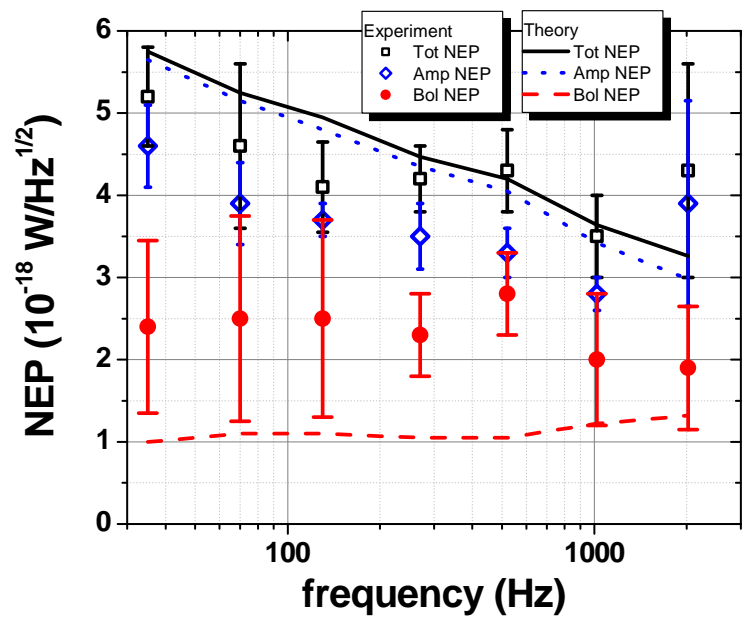

Fig. 22. Noise equivalent power spectrum from $35 \mathrm{~Hz}$ to $2 \mathrm{kHz}$. The bolometer NEP is at the level of $2^{*} 10^{-18} \mathrm{~W} / \mathrm{Hz}^{1 / 2}$, which agrees fairly well with what theory predicts. 


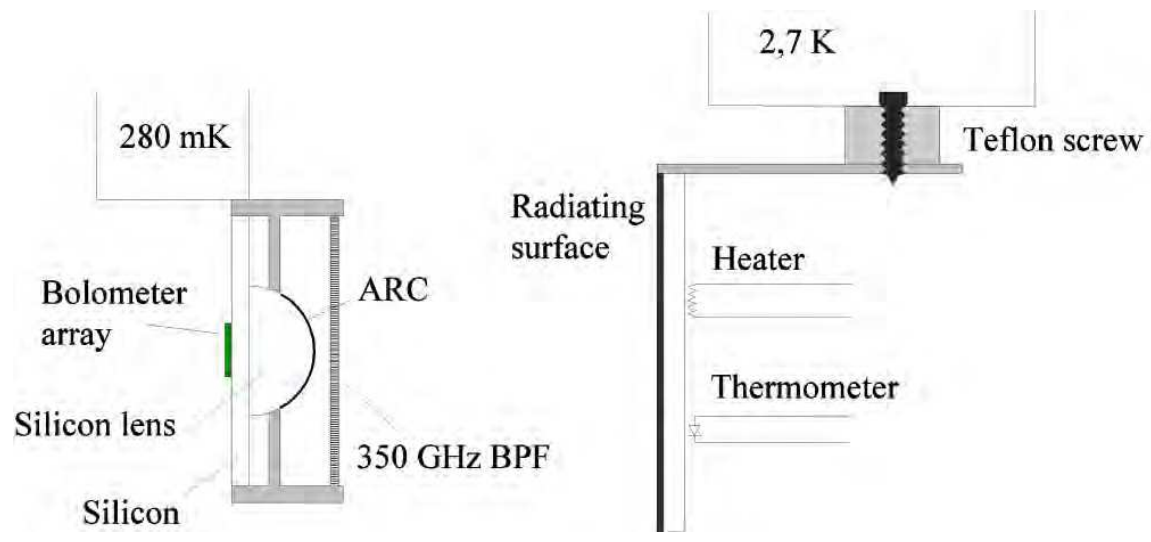

Fig. 23. Schematic view of optical response experiment. Here the silicon lens is covered with antireflection coating (ARC), incoming radiation pass through a band-pass filter for 350 GHZ (BPF). Thermal radiation source is equipped with heater and thermometer.

We measured the response to the microwave radiation emitted by a cryogenic blackbody radiation sources at the $100 \mathrm{mK}$ stage of dilution refrigerator. The radiation source was mounted on the $0.4 \mathrm{~K}$ stage; it consists of a Constantan foil with heater and thermometer. Using a backward wave oscillator spectrometer/reflectometer we measured the reflection of the foil $\mathrm{R}=0.70 \pm 0.05$ at $345 \mathrm{GHz}$. This value is different from zero reflectivity of blackbody and the actual emissivity of such source is $\mathrm{k}=0.30 \pm 0.05$. The response to heating of the emitter is presented in Fig. 24. The measured voltage response to temperature variations of the emitter is

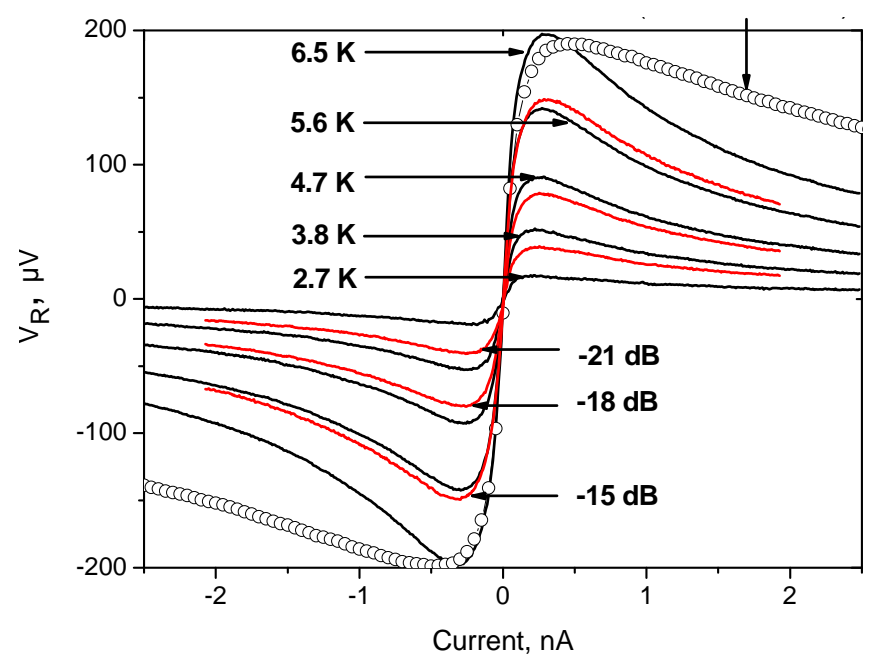

Fig. 24. Voltage response of the bolometer array to bath temperature changes 0.118-0.2 K (open circles), to variations of blackbody source temperature $2.7 \mathrm{~K}, 3.8 \mathrm{~K}, 4.7 \mathrm{~K}, 5.6 \mathrm{~K}, 6.5 \mathrm{~K}$ (solid lines) and also to $345 \mathrm{GHz}$ radiation from $\mathrm{BWO}$ with additional attenuation of $-21 \mathrm{~dB}$, $-18 \mathrm{~dB}$ and $-15 \mathrm{~dB}$ (dashed lines). 
$25 \mu \mathrm{V} / \mathrm{K}$. Taking into account the emissivity of foil and the root mean square voltage noise $0.38 \mu \mathrm{V}$ in the frequency range $0-40 \mathrm{~Hz}$, one can obtain the temperature sensitivity, which is $5 \mathrm{mK} \mathrm{rms}$. If we assume that the noise spectral density of the amplifier $10 \mathrm{nV} / \mathrm{Hz}^{1 / 2}$ dominates the total noise, we obtain a temperature sensitivity of $1.3^{*} 10^{-4} \mathrm{~K} / \mathrm{Hz}^{1 / 2}$. We can also calculate the power emitted by the heated foil using the Planck formula for central frequency $\mathrm{f}_{0}=345 \mathrm{GHz}$ and for bandwidth $\delta \mathrm{f}=100 \mathrm{GHz}$ of a cross-slot antenna.

At a temperature of $3 \mathrm{~K}$ we get $\Delta \mathrm{P}=3^{*} 10^{-14} \mathrm{~W}$, where $\mathrm{h}=6.626^{*} 10^{-34} \mathrm{~J}^{*} \mathrm{~s}$ is Planck's constant, $\mathrm{k}=1.38^{*} 10^{-23} \mathrm{~J} / \mathrm{K}$ is Boltzmann's constant, $\mathrm{f}$ is frequency and $\mathrm{K}$ is emissivity of radiation source. The voltage response to incoming power is thus $\mathrm{dV} / \mathrm{dP}=8^{*} 10^{8} \mathrm{~V} / \mathrm{W}$. For the experimentally measured noise of $10 \mathrm{nV} / \mathrm{Hz}^{1 / 2}$ this corresponds to an optical noise equivalent power $\mathrm{NEP}=2 * 10^{-17} \mathrm{~W} / \mathrm{Hz}^{1 / 2}$. In Fig.14 we show that the responses of the detector to variations of the power from a thermal radiation source and from a BWO are very similar, while the response to changes of the physical temperature of the sample is clearly very different. This difference can be due to suppression of the energy gap due to thermal heating.

\section{Conclusion}

A novel concept of the capacitively-coupled CEB has been developed for high performance cosmology instruments. The CEB concept is based on a unique combination of RF capacitive coupling of an absorber to the antenna through capacitance of the SIN tunnel junctions and direct electron cooling of the absorber by the same SIN tunnel junction. The noise properties of this device are improved considerably by decreasing the electron temperature. Direct electron cooling leads to a considerable increase of the saturation power due to removing incoming power from the sensitive nanoabsorber.

Different concepts of antenna coupled CEBs from single bolometer to 2D arrays of CEBs help to match with various electrodynamic environments for polarization sensitive measurements and wideband FTS spectroscopy. Flexibility in the dc connection of CEBs in combination with electronic cooling give the opportunity to work with any optical power load from $0.02 \mathrm{fW}$ to $10 \mathrm{pW}$ realizing bolometer noise less than photon noise of the incoming signal. CEB can be matched with any readout from low-ohmic SQUIDs to high-ohmic JFET.

\section{Acknowledgment}

The author thanks Dmitry Golubev, Phillip Mauskopf, Peter Day, Paolo de Bernardis and Mikhail Tarasov for useful discussions. The work was supported by SNSB, VR, STINT and SI Swedish grants.

\section{References}

Agulo, I., Kuzmin, L, Fominsky, M., and Tarasov, M. (2004) "Effective Electron Microrefrigeration by Superconductor-Insulator-Normal Metal Tunnel Junctions", Nanotechnology, 15, 224-228.

Agulo, I., Kuzmin, L, and Tarasov, M. (2005) "Attowatt sensitivity of the Cold-Electron Bolometer". Proc. of the 16th Int. Symp. On Space Terahertz Technol., pp 147-152, Gothenburg, Sweden.

Bakker, J., Van Kempen, H. \& Wyder, P. (1970). Phys. Letts. 31A, 290. 
Chattopadhay, G., Rice, F., Miller, D., LeDuc, H.G. \& Zmuidzinas, J. (1999). IEEE Microwave Guide Wave Lett., 9, 467.

Cho, A., Insights of the Decade: Precision Cosmology. Science, 330 p. 1615 (2010).

Day, P., LeDuc, H. G., Goldin, A., Dowell, C. D., \& Zmuidzinas, J. (2004). Farinfrared/summillimeter imager-polarimeter using distributed antenna-coupled transition edge sensors. SPIE 5498, pp 857-865.

Dolan, G. (1977). Offset masks for lift-off photoprocessing. Appl. Phys. Lett. 31, 337.

Golubev, D. \& Kuzmin, L. (2001). Nonequilibrium theory of the hot-electron bolometer with NIS tunnel junction. Journal of Applied Physics. Vol.89, pp. 6464-6472

Golwala, S., Johum, J. \& Sadoulet, B. (1997). Proc. of the 7 International Workshop on Low Temperature Detectors, Munich, pp 64-65.

K. Irwin. (1995). An Application of Electrothermal Feedback for High-Resolution Cryogenic Particle-Detection. Applied Physics Letters, 66, (1995) 1998

Kuzmin, L. \& Likharev, K. (1987). Direct Observation of the Correlated Discrete SingleElectron Tunneling, Jpn. J. Appl. Phys. 26 (suppl. 3), 1387 (1987).

Kuzmin, L. \& Safronov, M. (1988). Observation of Single-Electron Coulomb Phenomena in Edge Tunnel Junctions, Pis'ma Zh. Eksp. Teor. Fiz. JETP Lett. 48,250 (1988).

Kuzmin, L., Nazarov, Yu.V., Haviland, D.B., Delsing, P. \& Claeson, T. (1991). Coulomb Blockade and Incoherent Tunneling of Cooper Pair in Ultra-Small Junctions Affected by strong Quantum Fluctuations, Phys. Rev. Lett. Vol.67, 1161.

Kuzmin, L., \& Haviland, D.B. (1991). Observation of the Bloch Oscillations in an Ultrasmall Josephson Junction, Phys. Rev. Lett. Vol.67, 2890.

Kuzmin, L., Pashkin, Y. A., Zorin, A. \& Claeson, T. Linewidth of Bloch Oscillations in Small Josephson Junctions. Physica B, 203, 376, (1994).

Kuzmin, L. (1998) Capacitively Coupled Hot Electron Microbolometer as Perspective IR and Submm Wave Sensor, Proc. of the 9th Int. Symposium on Space Terahertz Technology, Pasadena, pp 99-103.

Kuzmin, L., Devyatov, I. \& Golubev, D. (1998). Cold-electron bolometer with electronic microrefrigeration and the general noise analysis. Proceeding of SPIE, v. 3465, SanDiego, pp. 193-199.

Kuzmin, L., Chouvaev. D., Tarasov, M., Sundquist, P., Willander, M., Claeson, T. (1999). On the concept of a normal metal hot-electron microbolometer for space applications. IEEE Trans. Appl. Supercond., v. 9, N 2, pp. 3186-3189.

Kuzmin, L. (2000). On the Concept of a Hot-Electron Microbolometer with Capacitive Coupling to the Antenna, Physica B: Condensed Matter, 284-288, 2129.

Kuzmin, L. (2001) Optimization of the hot-electron bolometer for space astronomy, SNED Proc., pp. 145-154, Naples,

Kuzmin, L., Golubev, D. (2002). On the concept of an optimal hot-electron bolometer with NIS tunnel junctions. Physica C 372-376, 378.

Kuzmin, L., Fominsky, M., Kalabukhov, A., Golubev D., \& Tarasov M. (2002). Capacitively Coupled Hot-Electron Nanobolometer with SIN Tunnel Junctions, Proc. of SPIE, 4855, pp 217-227.

Kuzmin, L. (2003). Superconducting cold-electron bolometer with proximity traps. Microelectronic Engineering. 69, p. 309.

Kuzmin, L. (2004) Ultimate Cold-Electron Bolometer with Strong Electrothermal Feedback, SPIE Proc., 5498, p 349, Glasgow. 
Kuzmin, L., Agulo I., Fominsky, M., Savin, A., Tarasov, M. (2004). Optimization of the electron cooling by SIN tunnel junctions, Superconductor Science \& Technology, 17, pp. $400-405$

Kuzmin, L. (2006). Ultimate Cold-Electron Bolometer with SIN Tunnel Junction \& Andreev Contact. Proc. of the 17th Int. Symp. on Space Terahertz Technol., pp 183-186.

Kuzmin, L. (2008a). Array of Cold-Electron Bolometers with SIN Tunnel Junctions for Cosmology Experiments. Journal of Physics: Conference Series (JPCS), 97, p. 012310.

Kuzmin, L. (2008b). A Parallel/Series Array of Cold-Electron Bolometers with SIN Tunnel Junctions for Cosmology Experiments, IEEE/CSC \& European Superconductivity News Forum, No. 3, pp 1-9.

Kuzmin, L. (2008c). Distributed Antenna-Coupled Cold-Electron Bolometers for Focal Plane Antenna, Proc. ISSTT conference, pp 154-158.

Kuzmin, L. (2008d). A Superconducting Cold-Electron Bolometer with SIS' and Josephson Tunnel Junctions, Journal of Low Temperature Physics, 151, pp. 292-297.

Kuzmin, L. (2011a). 2D Array of Cold-Electron Nanobolometers with Double Polarisation Cross-Dipole Antennas, accepted to Nanoscale Research Letters.

Kuzmin, L. (2011b). Advanced Shadow Evaporation Technique (ASHET) for Large Area Tunnel Junctions. US prepatent, Appl. No. 61/525242, filed August 19, 2011.

Kuzmin, L. (2011c). Two-Dimensional Array of Cold-Electron Bolometers for Ultrasensitive Polarization Measurements. Radiophisika. Izvestiya VUZov, т. LIV, N8-9, 607 (2011); Radiophysics and Quantum Electronics (2012).

Lee, A., Richards, P., Nam, S., Cabrera, B., Irwin, K. (1996). A superconducting bolometer with strong electrothermal feedback. Applied Physics Letters, 69, 1801.

Mather, J. C. (1982). Bolometer noise: nonequilibrium theory. Appl. Opt. 21,1125.

Masi, S. et al. (2006). Instrument, Method, Brightness and Polarization Maps from the 2003 flight of BOOMERanG, Astronomy and Astrophysics, 458,687-716, astro-ph/0507509

Nahum, M., Eiles, T.M. \& Martinis, J.M. (1994). Electronic microrefrigerator based on a NIS tunnel junction. Appl. Phys. Lett., 65, pp.3123-3125.

Otto, E., Tarasov, M., Kuzmin, L. (2007). Ti-TiO 2 -Al normal-insulator-superconductor tunnel junctions fabricated in direct- write technology, Supercond. Sci. Technol. 20 865-869.

Tarasov, M., Fominsky, M., Kalabukhov, A., \& Kuzmin, L. (2002). Experimental study of a normal-metal hot electron bolometer with capacitive coupling. JETP Letters, 76, pp. 507-510.

Tarasov, M., Kuzmin, L., Edelman, V., Kaurova, N., Fominsky, M. \& Ermakov, A. (2010). Optical response of a cold-electron bolometer array. JETP Letters, 92, pp. 416-420.

Tarasov, M., Kuzmin, L. \& Kaurova, N. (2009). Thin multilayer aluminum structures for superconducting devices. Instruments and Experimental Techniques, 52, pp. 877-881

Tarasov, M , Kuzmin, L.; Edelman, V.; Mahashabde S. and de Bernardis, P.(2011) Optical Response of a Cold-Electron Bolometer Array Integrated with a 345-GHz Cross-Slot Antenna, IEEE Transaction on Applied Superconductivity, 21, pp. 3635-3639.

Tran, H. \& Page, L. (2009). Optical elements for a CMBPol mission. Journal of Physics: Conference Series 155012007 


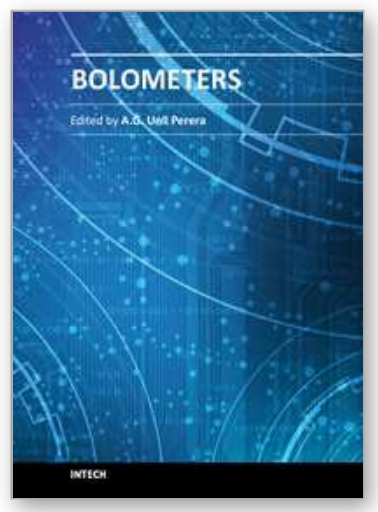

\author{
Bolometers \\ Edited by Prof. Unil Perera
}

ISBN 978-953-51-0235-9

Hard cover, 196 pages

Publisher InTech

Published online 09, March, 2012

Published in print edition March, 2012

Infrared Detectors and technologies are very important for a wide range of applications, not only for Military but also for various civilian applications. Comparatively fast bolometers can provide large quantities of low cost devices opening up a new era in infrared technologies. This book deals with various aspects of bolometer developments. It covers bolometer material aspects, different types of bolometers, performance limitations, applications and future trends. The chapters in this book will be useful for senior researchers as well as beginning graduate students.

\title{
How to reference
}

In order to correctly reference this scholarly work, feel free to copy and paste the following:

Leonid S. Kuzmin (2012). Cold-Electron Bolometer, Bolometers, Prof. Unil Perera (Ed.), ISBN: 978-953-510235-9, InTech, Available from: http://www.intechopen.com/books/bolometers/cold-eectron-bolometers

\section{INTECH}

open science | open minds

\author{
InTech Europe \\ University Campus STeP Ri \\ Slavka Krautzeka 83/A \\ 51000 Rijeka, Croatia \\ Phone: +385 (51) 770447 \\ Fax: +385 (51) 686166 \\ www.intechopen.com
}

\author{
InTech China \\ Unit 405, Office Block, Hotel Equatorial Shanghai \\ No.65, Yan An Road (West), Shanghai, 200040, China \\ 中国上海市延安西路65号上海国际贵都大饭店办公楼 405 单元 \\ Phone: +86-21-62489820 \\ Fax: $+86-21-62489821$
}


(C) 2012 The Author(s). Licensee IntechOpen. This is an open access article distributed under the terms of the Creative Commons Attribution 3.0 License, which permits unrestricted use, distribution, and reproduction in any medium, provided the original work is properly cited. 